\title{
Harmonicity and minimality of distributions on Riemannian manifolds via the intrinsic torsion
}

\author{
José Carmelo González-Dávila
}

\begin{abstract}
We consider a $q$-dimensional distribution as a section of the Grassmannian bundle $G_{q}\left(M^{n}\right)$ of $q$-planes and we derive, in terms of the intrinsic torsion of the corresponding $S(O(q) \times O(n-q))$-structure, the conditions that this map must satisfy in order to be critical for the functionals energy and volume. Using this it is shown that invariant Riemannian foliations of homogeneous Riemannian manifolds which are transversally symmetric determine harmonic maps and minimal immersions. In particular, canonical homogeneous fibrations on rank one normal homogeneous spaces or on compact irreducible 3 -symmetric spaces provide many examples of harmonic maps and minimal immersions of compact Riemannian manifolds.
\end{abstract}

\section{Introduction}

A $q$-dimensional distribution on an $n$-dimensional oriented Riemannian manifold $(M, g)$ can be view as a section of the Grassmannian bundle $\pi: G_{q}(M) \rightarrow M$ of $q$-planes in $T M$. Because $G_{q}(M)$ is diffeomorphic to the homogeneous fibre bundle $\mathcal{S O}(M) / S(O(q) \times O(n-q))$, where $\mathcal{S O}(M)$ is the the principal $S O(n)$ bundle of oriented orthonormal frames of $(M, g)$, it is endowed with a natural Riemannian metric $g^{K}$ related with $g$ known as the Kaluza-Klein metric [29]. It makes $\pi:\left(G_{q}(M), g^{K}\right) \rightarrow(M, g)$ a Riemannian submersion with totally geodesic fibres. If moreover $M$ is compact, since the fibres are also compact submanifolds, $\left(G_{q}(M), g^{K}\right)$ is a compact Riemannian manifold.

The energy functional of a distribution $\sigma$ is then defined as the energy of the $\operatorname{map} \sigma:(M, g) \rightarrow\left(G_{q}(M), g^{K}\right)$ and its volume $\operatorname{Vol}(\sigma)$ as the volume of the corresponding submanifold $\sigma(M)$ in $\left(G_{q}(M), g^{K}\right)$.

Mathematics Subject Classification (2010): Primary 58E20, 53C30; Secondary 53C42, 53C10. Keywords: Harmonic map, minimal immersion, homogeneous space, distribution, intrinsic torsion of a $G$-structure. 
The energy of a map $\sigma:\left(M, g_{M}\right) \rightarrow\left(N, g_{N}\right)$ between Riemannian manifolds, $M$ being compact and oriented, is the integral

$$
\mathcal{E}(\sigma)=\frac{1}{2} \int_{M} \operatorname{trace} L_{\sigma} d v_{g_{M}},
$$

where $L_{\sigma}$ is the $(1,1)$-tensor field determined by $\left(\sigma^{*} g_{N}\right)(X, Y)=g_{M}\left(L_{\sigma} X, Y\right)$, for all $X, Y \in$, where $\mathfrak{X}(M)$ is the Lie algebra of $C^{\infty}$ vector fields on $M$, and $d v_{g_{M}}$ denotes the volume form on $\left(M, g_{M}\right)$. The volume $\operatorname{Vol}(\sigma)$ of an immersion $\sigma: M \rightarrow$ $\left(N, g_{N}\right)$ is the volume of the Riemannian manifold $\left(M, \sigma^{*} g_{N}\right)$. If we choose a metric $g_{M}$ on $M$ then

$$
\operatorname{Vol}(\sigma)=\int_{M} \sqrt{\operatorname{det} L_{\sigma}} d v_{g_{M}} .
$$

The Euler-Lagrange equations of the corresponding variational problems give rise to the definition of the tension of a map and the mean curvature of an immersion. When $M$ is moreover closed, their vanishing characterizes harmonic maps and minimal immersions, respectively. We refer to [6], [7], and [25] for more information about the energy and volume functional.

We also consider $q$-dimensional oriented distributions on $M$ as sections of the Grassmannian bundle $\pi: G_{q}^{\text {or }}(M) \rightarrow M$ of oriented $q$-planes in $T M . G_{q}^{\text {or }}(M)$ is diffeomorphic to the homogeneous fibre bundle $\mathcal{S O}(M) /(S O(q) \times S O(n-q))$ and, with respect to the corresponding Kaluza-Klein metrics, $G_{q}^{\text {or }}(M)$ and $G_{q}(M)$ are locally isometric. Hence, the conditions characterizing harmonicity and minimality for oriented or unoriented distributions as maps into $G_{q}^{\text {or }}(M)$ or $G_{q}(M)$ are the same.

The Grassmannian bundle $G_{q}^{\text {or }}(M)$ can be identified with a subbundle of the bundle $\pi: \Lambda^{q}(M) \rightarrow M$ of all $q$-vectors on $M$. This allows us to see $G_{q}^{\text {or }}(M)$ as a Riemannian submanifold of $\Lambda^{q}(M)$ equipped with a Riemannian metric $g^{S}$, which generalizes the Sasaki metric of the tangent bundle; see [2]. We shall show in Theorem 3.2 that the Riemannian metrics $g^{K}$ and $g^{S}$ on $G_{q}^{\text {or }}(M)$ determine the same Riemannian structure. Hence, studies of the harmonicity for distributions made by viewing them as maps into $\left(G_{q}(M), g^{K}\right)$, as in [5] and [28], among others; or for oriented distributions by viewing them as maps into $\left(G_{q}^{\text {or }}(M), i^{*} g^{S}\right)$, as in [4], [8], [20] and [22], yield the same theory.

In terms of $G$-structures, $q$-dimensional distributions are in one-to-one correspondence with $S(O(q) \times O(n-q))$-reductions of the $S O(n)$-bundle $\pi: \mathcal{S O}(M) \rightarrow M$, see [23]. In the present work, we analyze the energy functional of a distribution $\sigma$ considered as a map $\sigma:(M, \tilde{g}) \rightarrow\left(G_{q}(M), g^{K}\right)$, where $\tilde{g}$ is an arbitrary metric on $M$, and we show the central role played by the intrinsic torsion of the $S(O(q) \times$ $O(n-q))$-structure in the characterization of critical points for the energy and the volume functional (Theorem 3.4).

The use of the intrinsic torsion has made possible deeper study of the harmonicity of $G$-structures (see [10] and [11]). Here, we focus attention on Riemannian homogeneous manifolds $(M=G / K, g)$ equipped with a $G$-invariant integrable distribution $\mathcal{F}$ whose transverse geometry is locally modeled on a symmetric space, called a transversally symmetric invariant foliation, and, using tools developed for 
the intrinsic torsion of $S(O(q) \times O(n-q))$-structures, we show that $\mathcal{F}$ is a harmonic distribution, a critical point of the energy functional restricted to $\Gamma^{\infty}\left(G_{q}(M)\right)$, and a minimal immersion into the Grassmannian bundle $\left(G_{q}(M), g^{K}\right)$. When $\mathcal{F}$ is moreover a Riemannian foliation, see [21], we will be able to show that it determines a harmonic map $\sigma:(M, g) \rightarrow\left(G_{q}(M), g^{K}\right)$ (Theorem 4.2). This yields new examples of harmonic maps between compact Riemannian manifolds.

A standard way of constructing transversally symmetric invariant foliations on $(M=G / K, g)$ consists in taking as the leaves the fibres of a homogeneous fibration

$$
F=L / K \rightarrow M=G / K \stackrel{\pi}{\rightarrow} N=G / L: g K \mapsto g L,
$$

where $(G, L)$ is a symmetric pair. In fact, if $G$ is semisimple, any of these invariant foliations becomes precisely a homogeneous fibration of the type above. Using a different method, when $G, L$ and $K$ are compact Lie groups and the metric $g$ is a suitable homogeneous Riemannian metric, known as binormal, the harmonicity of the transversally symmetric fibration is proved in [5]. Here, we extend this result not only to harmonicity but also to minimality of homogeneous fibrations with respect to any invariant metric on the total space $M=G / K$.

Compact rank one normal homogeneous spaces $([13],[26])$ and irreducible 3symmetric spaces of types $A_{3} I I$ and $A_{3} I I I$ ([9], [12], [14], [27]) admit canonical fibrations over (irreducible) symmetric spaces of compact type which are constructed in this way. On compact rank one homogeneous spaces, they are all the generalized Hopf fibrations (see Chapter $9 \mathrm{H}$ of [1]) together with the two exceptional fibrations

$$
\mathbb{R} P^{5} \rightarrow B^{13} \rightarrow \mathbb{C} P^{4}, \quad \mathbb{R} P^{3} \rightarrow W^{7} \rightarrow \mathbb{C} P^{2},
$$

where $B^{13}$ and $W^{7}$ denote respectively the Berger and Wilking spaces [26]. On compact irreducible 3-symmetric spaces, we give the complete list of the canonical fibrations and we show that for any homogeneous Riemannian metric on the total space, all these fibrations determine harmonic maps and minimal immersions into their Grassmannian bundles (Theorems 5.3, 5.4, and 5.6).

\section{Intrinsic torsion of distributions}

Let $\pi: G_{q}(M)=\bigcup_{x \in M} G_{q}\left(T_{x} M\right) \rightarrow M$ be the Grassmannian bundle of the $q$-dimensional linear subspaces in the tangent bundle $T M$ of a $n$-dimensional oriented Riemannian manifold $(M, g)$. For each $x \in M$, the fibre $G_{q}\left(T_{x} M\right)$ is a $(n-q) q$-dimensional compact homogeneous manifold diffeomorphic to $G_{q}\left(\mathbb{R}^{n}\right)=$ $S O(n) / S(O(q) \times O(n-q))$.

Let $\pi_{S O(n)}: \mathcal{S O}(M) \rightarrow M$ be the principal $S O(n)$-bundle of oriented orthonormal frames of $(M, g)$. A point $p$ of $\mathcal{S O}(M)$ is a pair $\left(x ; p_{1}, \ldots, p_{n}\right)$ where $x \in M$ and $\left\{p_{1}, \ldots, p_{n}\right\}$ is an oriented and orthonormal basis of $\left(T_{x} M, g_{x}\right)$ and the action of $S O(n)$ on the right on $\mathcal{S O}(M)$ is given by $p \cdot a=\left(x ; a p_{1}, \ldots, a p_{n}\right)$, where $a p_{i}=a_{i}^{j} p_{j}$ and $a=\left(a_{j}^{i}\right) \in S O(n)$.

Factoring the map $\mathcal{S O}(M) \rightarrow G_{q}(M), p=\left(x ; p_{1}, \ldots, p_{n}\right) \mapsto \mathbb{R}\left\{p_{1}, \ldots, p_{q}\right\}$ by the Lie subgroup $S(O(q) \times O(n-q))$ of $S O(n), G_{q}(M)$ is diffeomorphic to the 
orbit space $\mathcal{S O}(M) / S(O(q) \times O(n-q))$ and so, it is naturally isomorphic to the associated bundle $\mathcal{S O}(M) \times{ }_{S O(n)} G_{q}\left(\mathbb{R}^{n}\right)$ via the map

$$
[(p, a S(O(q) \times O(n-q)))] \mapsto p \cdot a S(O(q) \times O(n-q)) .
$$

Moreover, the orbit map $\rho: \mathcal{S O}(M) \rightarrow \mathcal{S O}(M) / S(O(q) \times O(n-q))$ is a principal $S(O(q) \times O(n-q))$-bundle and we have $\pi_{S O(n)}=\pi \circ \rho$. Hence, each $\sigma \in \Gamma^{\infty}\left(G_{q} M\right)$ determines a reduction of $\mathcal{S O}_{\sigma}(M) \subset \mathcal{S O}(M)$ to $S(O(q) \times O(n-q))$ where

$$
\mathcal{S O}_{\sigma}(M)=\left\{\left(x ; p_{1}, \ldots, p_{n}\right) \in \mathcal{S O}(M) \mid \sigma(x)=\mathbb{R}\left\{p_{1}, \ldots, p_{q}\right\}\right\}
$$

and conversely. Then there is a one-to-one correspondence between the set of $S(O(q) \times O(n-q))$-structures and the manifold $\Gamma^{\infty}\left(G_{q}(M)\right)$ of all $q$-dimensional distributions of $(M, g)$. Moreover, the pair $\left(\mathcal{V}_{\sigma}=\sigma, \mathcal{H}_{\sigma}=\sigma^{\perp}\right)$, where $\sigma^{\perp}$ is the orthogonal distribution of $\sigma$ on $(M, g)$, determines a Riemannian almost-product $(A P)$ structure, i.e., an orthogonal $(1,1)$-tensor field $P_{\sigma}$ on $(M, g)$ with $P_{\sigma}^{2}=\mathrm{Id}$ and $P_{\sigma} \neq \pm \mathrm{Id}$. The vertical and horizontal distributions $\mathcal{V}_{\sigma}$ and $\mathcal{H}_{\sigma}$ are the corresponding \pm 1 -eigendistributions of $P_{\sigma}$.

In similar way, if we consider the Grassmannian bundle $G_{q}^{\text {or }}(M)$ of the $q$-dimensional oriented linear subspaces of $T M$, with fibres diffeomorphic to the Grassmannian manifold $G_{q}^{\text {or }}\left(\mathbb{R}^{n}\right)=S O(n) / S O(q) \times S O(n-q)$, one obtains that each $S O(q) \times S O(n-q)$-structure corresponds with a $q$-dimensional oriented distribution.

Each of the Grassmannian manifolds $G_{q}\left(\mathbb{R}^{n}\right)$ and $G_{q}^{\text {or }}\left(\mathbb{R}^{n}\right)$ is a Riemannian symmetric space equipped with the normal homogeneous Riemannian metric that, up to a scalar, is induced on $\mathfrak{s o}(n)$ by the inner product $\langle X, Y\rangle=-\frac{1}{2}$ trace $X Y$. The associated orthogonal symmetric Lie algebra is the pair $(\mathfrak{s o}(n), \mathfrak{s}), \mathfrak{s}$ being the involutive automorphism $\mathfrak{s}(X)=P X P$ of $\mathfrak{s o}(n)$, given by conjugation by the matrix

$$
P=\left(\begin{array}{cc}
I_{q} & 0 \\
0 & -I_{n-q}
\end{array}\right) .
$$

Then $\mathfrak{s o}(n)=(\mathfrak{s o}(q) \oplus \mathfrak{s o}(n-q)) \oplus \mathfrak{m}$ is a reductive decomposition, where $\mathfrak{m}$ is the -1-eigenspace of $\mathfrak{s}$, the subspace of $\mathfrak{s o}(n)$ generated by the matrices $\left\{B_{j}^{a}=\right.$ $\left.E_{q+j, a}-E_{a, q+j}: a=1, \ldots, q ; j=1, \ldots, n-q\right\}$. Here, $E_{i j}$ denotes the $n \times n$-matrix with a 1 in the $i$ th row and $j$ th column, and all of other entries equal to 0 . These matrices form an orthonormal basis on $\mathfrak{m}$ with respect to $\langle\cdot, \cdot\rangle$.

Let $\mathfrak{s o}(M)$ be the subbundle of $\operatorname{End}(T M)$ of endomorphisms skew-symmetric with respect to $g$. It may be expressed as $\mathcal{S O}_{\sigma}(M) \times_{S(O(q) \times O(n-q))} \mathfrak{s o}(n)$, for each $\sigma \in \Gamma^{\infty}\left(G_{q}(M)\right)$. We consider the subbundle $\mathfrak{m}_{\sigma}=\mathcal{S O}_{\sigma}(M) \times_{S(O(q) \times O(n-q))} \mathfrak{m}$. Then $\mathfrak{m}_{\sigma}$ consists of those skew-symmetric endomorphisms $A$ of the tangent bundle such that $A P_{\sigma}=-P_{\sigma} A$, where $P_{\sigma}$ is the AP-structure associated to $\sigma$.

An oriented Riemannian manifold $(M, g)$ equipped with a $G$-structure $\sigma$ admits a unique G-connection $\nabla^{\sigma}=\nabla-\xi_{\sigma}$, the minimal connection, where $\nabla$ is the Levi-Civita connection of $(M, g)$, and the intrinsic torsion $\xi_{\sigma}$ of the G-structure belongs to $T^{*} M \otimes \mathfrak{m}_{\sigma}$, [3]. Next we determine the minimal connection and intrinsic 
torsion of an oriented distribution in terms of the corresponding Riemannian APstructure, using the identification of $S O(q) \times S O(n-q)$-structures as $q$-dimensional oriented distributions. Because $S O(q) \times S O(n-q)$ is the connected component of the identity of $S(O(q) \times O(n-q))$, these are also the minimal connection and the intrinsic torsion of the corresponding unoriented distribution.

Proposition 2.1. The minimal connection of a q-dimensional distribution $\sigma$ coincides with the Schouten connection $\nabla^{\sigma}=\nabla-\xi_{\sigma}$, where the intrinsic torsion $\xi_{\sigma}$ is given by

$$
\left(\xi_{\sigma}\right)_{X} Y=-\frac{1}{2} P_{\sigma}\left(\nabla_{X} P_{\sigma}\right) Y, \quad X, Y \in \mathfrak{X}(M) .
$$

Proof. This follows from the identities

$$
\nabla^{\sigma} P_{\sigma}=\nabla^{\sigma} g=0 \text { and } P_{\sigma} \circ\left(\xi_{\sigma}\right)_{X}=-\left(\xi_{\sigma}\right)_{X} \circ P_{\sigma}, \quad \text { for all } X \in \mathfrak{X}(M) .
$$

Let

$$
p_{1}=\frac{1}{2}(\mathrm{Id}+P): \mathrm{TM} \rightarrow \mathcal{V} \quad \text { and } \quad p_{2}=\frac{1}{2}(\mathrm{Id}-P): \mathrm{TM} \rightarrow \mathcal{H}
$$

be the canonical projections. Then, from (2.1), the intrinsic torsion $\xi$ of $\sigma$ is determined by

$$
\begin{aligned}
& \xi_{U} V=p_{2}\left(\nabla_{U} V\right), \quad \xi_{U} X=p_{1}\left(\nabla_{U} X\right), \\
& \xi_{X} U=p_{2}\left(\nabla_{X} U\right), \quad \xi_{X} Y=p_{1}\left(\nabla_{X} Y\right) .
\end{aligned}
$$

Here, and in what follows, $U, V$ and $W$ denote elements of $\mathcal{V} ; X, Y$ and $Z$ denote elements of $\mathcal{H}$ and, when a distribution $\sigma$ is fixed, we omit the subscript and we simply write $\xi, P, \mathcal{H}, \mathcal{V}, \ldots$ The second fundamental forms (symmetric tensors) $h_{\mathcal{V}}: \mathcal{V} \times \mathcal{V} \rightarrow \mathcal{H}$ and $h_{\mathcal{H}}: \mathcal{H} \times \mathcal{H} \rightarrow \mathcal{V}$, and the integrability tensors (skew-symmetric tensors) $A_{\mathcal{V}}: \mathcal{V} \times \mathcal{V} \rightarrow \mathcal{H}$ and $A_{\mathcal{H}}: \mathcal{H} \times \mathcal{H} \rightarrow \mathcal{V}$ are defined in terms of $\xi$ by the following formulas:

$$
\begin{array}{ll}
h_{\mathcal{V}}(U, V)=\frac{1}{2}\left(\xi_{U} V+\xi_{V} U\right), & A_{\mathcal{V}}(U, V)=\frac{1}{2}\left(\xi_{U} V-\xi_{V} U\right), \\
h_{\mathcal{H}}(X, Y)=\frac{1}{2}\left(\xi_{X} Y+\xi_{Y} X\right), & A_{\mathcal{H}}(X, Y)=\frac{1}{2}\left(\xi_{X} Y-\xi_{Y} X\right) .
\end{array}
$$

In view of the identities $A_{\mathcal{V}}(U, V)=\frac{1}{2} p_{2}[U, V]$ and $A_{\mathcal{H}}(X, Y)=\frac{1}{2} p_{1}[X, Y]$, the distribution $\mathcal{V}$ (resp., $\mathcal{H}$ ) is integrable, i.e., it determines a foliation, if and only if $A_{\mathcal{V}}=0$ (resp., $A_{\mathcal{H}}=0$ ). The distribution $\mathcal{V}($ resp., $\mathcal{H})$ is said to be geodesic if $h_{\mathcal{V}}=0$ (resp., $h_{\mathcal{H}}=0$ ). This means that all geodesics with initial vector in $\mathcal{V}$ (resp. $\mathcal{H}$ ) remain in $\mathcal{V}$ (resp. $\mathcal{H}$ ) for all time. Note that if $\xi$ is totally skewsymmetric, i.e., $\xi_{A} A=0$ for all $A \in T M$, then $\xi=0$.

A foliation on $(M, g)$ is said to be Riemannian if each geodesic orthogonal to a leaf at some point remains orthogonal to every leaf it meets. Tbus, a Riemannian foliation is determined by an integrable distribution $\mathcal{V}$ whose orthogonal distribution $\mathcal{H}$ is totally geodesic, or equivalently, has intrinsic torsion $\xi$ satisfying $\xi_{U} V=\xi_{V} U$ and $\xi_{X} Y=-\xi_{Y} X$. Locally the leaves are the fibres of a Riemannian submersion. 


\section{Harmonic and minimal distributions}

The Kaluza-Klein metric $g^{K}$ relative to $(g,\langle\cdot, \cdot\rangle)$ on the Grassmannian bundles $G_{q}(M)$ and $G_{q}^{\text {or }}(M)$ makes the projection $\pi$ onto $(M, g)$ a Riemannian submersion with totally geodesic fibres. It is defined on $G_{q}(M)$ - in similar way on $G_{q}^{\text {or }}(M)$ as follows, [29]: let $T G_{q}(M)=\mathcal{V} \oplus \mathcal{H}$, where

$$
\mathcal{V}=\operatorname{Ker} \pi_{*}=\rho_{*}\left(\operatorname{Ker}\left(\pi_{S O(n)}\right)_{*}\right), \quad \mathcal{H}=\rho_{*}(\operatorname{Ker} \omega),
$$

and $\omega$ is the $\mathfrak{s o}(n)$-valued connection form of the Levi-Civita connection on $\mathcal{S O}(M)$. Then, for each $p \in \mathcal{S O}(M)$, a vector in $\mathcal{V}_{\rho(p)}$ may be written as $\rho_{* p} A_{p}^{*}$, for some $A \in \mathfrak{m}, A^{*}$ being the fundamental vector field on $\mathcal{S O}(M)$.

We consider the canonical bundle $\mathfrak{m}_{\mathcal{S O}(M)}=\mathcal{S O}(M) \times_{S(O(q) \times O(n-q))} \mathfrak{m}$ associated to $\rho$ with fibre $\mathfrak{m}$. Then the map $\iota: \mathcal{V} \rightarrow \mathfrak{m}_{\mathcal{S O}(M)}, \rho_{* p} A_{p}^{*} \mapsto[(p, A)]$, is a vector bundle isomorphism, which may be extended to $\mathcal{K}: T G_{q}(M) \rightarrow \mathfrak{m}_{\mathcal{S O}(M)}$ by saying that $\mathcal{K}(\eta)=0$ for all $\eta \in \mathcal{H}$ and $\mathcal{K}(\eta)=\iota(\eta)$ for all $\eta \in \mathcal{V}$. Denoting also by $\langle\cdot, \cdot\rangle$ the fibre metric on $\mathfrak{m}_{\mathcal{S O}(M)}$ induced by the inner product on $\mathfrak{m}$, the metric $g^{K}$ is then characterized by

$$
g^{K}\left(\eta_{1}, \eta_{2}\right)=g\left(\pi_{*} \eta_{1}, \pi_{*} \eta_{2}\right)+\left\langle\mathcal{K}\left(\eta_{1}\right), \mathcal{K}\left(\eta_{2}\right)\right\rangle .
$$

With respect to the corresponding Kaluza-Klein metrics on $G_{q}^{\text {or }}(M)$ and $G_{q}(M)$, the projection $p_{r}: G_{q}^{\text {or }}(M) \rightarrow G_{q}(M),[(p, a S O(q) \times S O(n-q))] \mapsto[(p, a S(O(q) \times$ $O(n-q)))]$, is a local isometry.

On the other hand, we can think of $G_{q}^{\text {or }}(M)$ as a subbundle of the bundle $\pi: \Lambda^{q}(M) \rightarrow M$ of all skew-symmetric contravariant tensors of order $q$; or briefly, of all $q$-vectors on $M$ (see [8]). This allows us to see $G_{q}^{\text {or }}(M)$ as a Riemannian submanifold of $\Lambda^{q}(M)$ equipped with a Riemannian metric $g^{S}$, generalizing the Sasaki metric of the tangent bundle, see [2]. For $\sigma \in \Lambda^{q}(M)$ and $\eta_{1}, \eta_{2} \in T_{\sigma} \Lambda^{q}(M)$, $g^{S}$ is defined by

$$
g^{S}\left(\eta_{1}, \eta_{2}\right)=g\left(\pi_{*} \eta_{1}, \pi_{*} \eta_{2}\right)+g\left(K\left(\eta_{1}\right), K\left(\eta_{2}\right)\right),
$$

where $g$ also denotes the natural extension of the metric $g$ of $M$ to $q$-vectors and $K: T \Lambda^{q}(M) \rightarrow \Lambda^{q}(M)$ is the connection map on $\Lambda^{q}(M)$ induced by the LeviCivita connection $\nabla$ of $g$. If $\sigma$ is a smooth section, $\sigma \in \Gamma^{\infty}\left(\Lambda^{q}(M)\right)$, one obtains that $K\left(\sigma_{*} X\right)=\nabla_{X} \sigma$, for all $X \in \mathfrak{X}(M)$. Then, from (3.1), we get

$$
\left(\sigma^{*} g^{S}\right)(X, Y)=g(X, Y)+g\left((\nabla \sigma)^{\mathrm{t}} \circ(\nabla \sigma)(X), Y\right),
$$

where $\nabla \sigma$ is the map $\nabla \sigma: \mathfrak{X}(M) \rightarrow \Gamma^{\infty}\left(\Lambda^{q}(M)\right),(\nabla \sigma)(X)=\nabla_{X} \sigma$, and $(\nabla \sigma)^{\mathrm{t}}$ : $\Gamma^{\infty}\left(\Lambda^{q}(M)\right) \rightarrow \mathfrak{X}(M)$ is the adjoint operator of $\nabla \sigma$ with respect to $g$ defined by

$$
g\left((\nabla \sigma)^{\mathrm{t}} \varphi, X\right)=g\left(\varphi, \nabla_{X} \sigma\right), \quad \varphi \in \Gamma^{\infty}\left(\Lambda^{q}(M)\right), X \in \mathfrak{X}(M) .
$$

Let $\Sigma_{q}^{\text {or }}\left(\mathbb{R}^{n}\right)$ be the set of all decomposable $q$-vectors $\sigma$ of $\mathbb{R}^{n}$ such that $\|\sigma\|=1$. Then $\sigma$ may be written as $\sigma=e_{1} \wedge \cdots \wedge e_{q}$ where $\left\{e_{1}, \ldots, e_{q}\right\}$ are the first $q$ vectors of a positive orthonormal basis $\left\{e_{i}\right\}_{i=1}^{n}$ of $\mathbb{R}^{n}$. The natural action of $S O(n)$ on $\mathbb{R}^{n}$ determines a transitive action on $\Sigma_{q}^{\text {or }}\left(\mathbb{R}^{n}\right)$ given by $a \cdot \sigma=a e_{1} \wedge \cdots \wedge a e_{q}, a \in S O(n)$. Write $\sigma_{0}=u_{1} \wedge \cdots \wedge u_{q}$, where $\left\{u_{i}\right\}_{i=1}^{n}$ is the canonical basis of $\mathbb{R}^{n}$. Then, we have: 
Lemma 3.1. The map $\Phi: G_{q}^{\text {or }}\left(\mathbb{R}^{n}\right) \rightarrow \Sigma_{q}^{\text {or }}\left(\mathbb{R}^{n}\right), a(S O(q) \times S O(n-q)) \mapsto a \cdot \sigma_{0}$, is an isometry, where $\Sigma_{q}^{\mathrm{or}}\left(\mathbb{R}^{n}\right)$ is considered as Riemannian submanifold of $\Lambda^{q}\left(\mathbb{R}^{n}\right)$.

Proof. The tangent space $T_{\sigma} \Sigma_{q}^{\text {or }}\left(\mathbb{R}^{n}\right)$ at $\sigma=e_{1} \wedge \cdots \wedge e_{q}$ is the subspace of $\Lambda^{q}\left(\mathbb{R}^{n}\right)$ generated by

$$
\left\{\sigma_{j}^{a}=(-1)^{a+1} e_{q+j} \wedge e_{1} \wedge \cdots \wedge \hat{e}_{a} \wedge \cdots \wedge e_{q} \mid a=1, \ldots, q ; j=1, \ldots, n-q\right\}
$$

(see Section 3 in [8]), where $\left\{e_{q+1}, \ldots, e_{n}\right\}$ are chosen such that they complete $\left\{e_{1}, \ldots, e_{q}\right\}$ to a positive orthonormal basis of $\mathbb{R}^{n}$ and $\hat{e}_{a}$ means that $e_{a}$ is omitted. With respect to the induced metric on $\Sigma_{q}^{\text {or }}\left(\mathbb{R}^{n}\right) \subset \Lambda^{q}\left(\mathbb{R}^{n}\right)$, the $\left\{\sigma_{j}^{a}\right\}$ form an orthonormal basis of $T_{\sigma} \Sigma_{q}^{\text {or }}\left(\mathbb{R}^{n}\right)$. Moreover, this metric is $S O(n)$-invariant and $\Sigma_{q}^{\text {or }}\left(\mathbb{R}^{n}\right)$ becomes a homogeneous Riemannian manifold. Hence we must show that for the corresponding inner products, $\Phi_{* o}: \mathfrak{m} \rightarrow T_{\sigma_{o}} \Sigma_{q}^{\text {or }}\left(\mathbb{R}^{n}\right)$ is a linear isometry, or equivalently, $\Phi_{* o} B_{j}^{a}=\left(\sigma_{0}\right)_{j}^{a}$, where $o$ is the origin of the homogeneous space $S O(n) / S O(q) \times S O(n-q)$. Now, we have

$$
\Phi_{* o} B_{j}^{a}=\left.\frac{d}{d t}\right|_{t=0} e^{t B_{j}^{a}} \cdot \sigma_{0}=\sum_{b=1}^{q}\left(u_{1} \wedge \cdots \wedge \frac{d}{d t}_{\mid t=0} e^{t B_{j}^{a}} u_{b} \wedge \cdots \wedge u_{q}\right) .
$$

Then, taking into account that $e^{t B_{j}^{a}} u_{a}=\cos t u_{a}+\sin t u_{q+j}$ and $e^{t B_{j}^{a}} u_{b}=u_{b}$, for $b \neq a$, it follows that

$$
\Phi_{* o} B_{j}^{a}=(-1)^{a+1} \frac{d}{d t}{ }_{\mid t=0}\left(\cos t u_{a}+\sin t u_{q+j}\right) \wedge u_{1} \wedge \cdots \wedge \hat{u}_{a} \wedge \cdots \wedge u_{q}=\left(\sigma_{0}\right)_{j}^{a} .
$$

Let $\Sigma_{q}^{\mathrm{or}}(M)=\bigcup_{x \in M} \Sigma_{q}^{\mathrm{or}}\left(T_{x} M\right)$ be the subbundle of $\Lambda^{q}(M)$ comprising unit norm decomposable forms. It can be expressed as the associated bundle $\mathcal{S O}(M)$ $\times_{S O(n)} \Sigma_{q}^{\text {or }}\left(\mathbb{R}^{n}\right)$ of $\pi_{S O(n)}$. Then, we have:

Theorem 3.2. The map $\tilde{\Phi}:\left(G_{q}^{\text {or }}(M), g^{K}\right) \rightarrow\left(\Sigma_{q}^{\text {or }}(M), i^{*} g^{S}\right)$ given by

$$
\tilde{\Phi}([(p, a S O(q) \times S O(n-q))])=[(p, \Phi(a S O(q) \times S O(n-q)))],
$$

is an isometry.

Proof. By Lemma 3.1, the proof reduces to showing that $\tilde{\Phi}_{*}\left(X^{H}\right)$ is horizontal, where $X^{H}$ denotes the horizontal lift in $G_{q}^{\text {or }}(M)$ of $X \in \mathfrak{X}(M)$. Let $\alpha=\alpha(t)$ be a curve in $M$ and let $\left\{E_{1}, \ldots, E_{n}\right\}$ be an oriented orthonormal frame field along $\alpha$ obtained by parallel translation with respect to the Levi-Civita connection. Then $t \mapsto\left(\alpha(t) ; E_{1}(t), \ldots, E_{n}(t)\right)$ gives a horizontal curve in $\mathcal{S O}(M)$ and, via $\rho$, the map $t \mapsto U(t)=\mathbb{R}\left\{E_{1}(t), \ldots, E_{q}(t)\right\}$ determines a curve in $G_{q}^{\text {or }}(M)$ tangent to the horizontal distribution $\mathcal{H}$. Its image under $\tilde{\Phi}$ is the curve $t \mapsto \sigma(t)=$ $E_{1}(t) \wedge \cdots \wedge E_{q}(t)$ in $\Sigma_{q}^{\text {or }}(M)$. Since

$$
\frac{\nabla \sigma(t)}{d t}=\sum_{a=1}^{q} E_{1}(t) \wedge \cdots \wedge \frac{\nabla E_{a}(t)}{d t} \wedge \cdots \wedge E_{q}(t)
$$

$\sigma(t)$ is parallel along $\alpha$, or equivalently, a horizontal lift of $\alpha$. Hence, $\tilde{\Phi}$ sends horizontal lifts of a curve $\alpha$ in $M$ to horizontal lifts of the same curve $\alpha$. 
Remark 3.3. Denote by $\Sigma_{q}\left(\mathbb{R}^{n}\right)$ the orbit space $\Sigma_{q}^{\text {or }}\left(\mathbb{R}^{n}\right) / \pm 1$ and by $\Sigma_{q}(M)$ the associated bundle $\mathcal{S O}(M) \times_{S O(n)} \Sigma_{q}\left(\mathbb{R}^{n}\right)$ of $\pi_{S O(n)}$. Then $\tilde{\Phi}$ induces an isometry between $\left(G_{q}(M), g^{K}\right)$ and $\left(\Sigma_{q}(M), i^{*} g^{S}\right)$, where $i^{*} g^{S}$ also denotes the metric on $\Sigma_{q}(M)$ making $\Sigma_{q}^{\text {or }}(M) \rightarrow \Sigma_{q}(M),[(p, \sigma)] \mapsto[(p,[\sigma])]$, a local isometry.

Under the identification above, $G_{q}^{\text {or }}(M)$ is the subbundle of $\Lambda^{q}(M)$ comprising unit norm decomposable forms, and each $q$-dimensional oriented distribution $\sigma \in \Gamma^{\infty}\left(G_{q}^{\text {or }}(M)\right)$ can be considered as a global smooth section of the tensor bundle $\Lambda^{q}(M)$. It can be expressed locally as $\sigma=E_{1} \wedge \cdots \wedge E_{q}$, where $\left\{E_{1}, \ldots, E_{n}\right\}$ is a positive orthonormal local frame such that the $E_{1}, \ldots, E_{q}$ span $\sigma$ and the $E_{q+1}, \ldots, E_{n}$ span $\sigma^{\perp}$, section of $G_{n-q}^{\text {or }}(M)$. Such a local frame $\left\{E_{1}, \ldots, E_{q} ; E_{q+1}, \ldots, E_{n}\right\}$ will be called a local frame adapted to the distribution.

In what follows, the following convention for the indices is used: $i \in\{1, \ldots, n\}$, $a, b, c, \cdots \in\{1, \ldots, q\}$ and $j, k, l, \cdots \in\{1, \ldots, n-q\}$. Then, with respect to an adapted local frame and using the summation convention, we have

$$
\nabla_{X} \sigma=E_{1} \wedge \cdots \wedge \nabla_{X} E_{a} \wedge \cdots \wedge E_{q}=g\left(\xi_{X} E_{a}, E_{q+j}\right) \sigma_{j}^{a} .
$$

Since $g\left(\sigma_{j}^{a}, \sigma_{k}^{b}\right)=\delta_{j}^{k} \delta_{a}^{b}$, taking into account (2.2) we obtain

$$
g\left(\nabla_{X} \sigma, \nabla_{Y} \sigma\right)=g\left(\xi_{X} E_{a}, E_{q+j}\right) g\left(\xi_{Y} E_{a}, E_{q+j}\right)=\frac{1}{2} g\left(\xi_{X}, \xi_{Y}\right) .
$$

If $M$ is compact and oriented, the energy functional of an oriented distribution $\sigma$ is defined as the energy $\mathcal{E}(\sigma)$ of the map $\sigma:(M, g) \rightarrow\left(G_{q}^{\text {or }}(M), g^{K}\right) \cong$ $\left(\Sigma_{q}^{\mathrm{or}}(M), i^{*} g^{S}\right)$. Using (3.2) and (3.4), the tensor field $L_{\sigma}$ defined in Section 1 can be expressed as

$$
L_{\sigma}=\mathrm{Id}+\frac{1}{2} \xi_{\sigma}^{t} \circ \xi_{\sigma},
$$

where $\xi_{\sigma}$ is considered as the $C^{\infty}(M)$-map $\xi_{\sigma}: \mathfrak{X}(M) \rightarrow \Gamma^{\infty}\left(\mathfrak{m}_{\sigma}\right), \xi_{\sigma} X=\left(\xi_{\sigma}\right)_{X}$. Hence, the energy $\mathcal{E}(\sigma)$ and the volume $\operatorname{Vol}(\sigma)$ of $\sigma$ are given in terms of the intrinsic torsion by

$$
\mathcal{E}(\sigma)=\frac{n}{2} \operatorname{Vol}(M)+\frac{1}{4} \int_{M}\left\|\xi_{\sigma}\right\|^{2} d v_{g}, \quad \operatorname{Vol}(\sigma)=\int_{M} \sqrt{\operatorname{det}\left(\operatorname{Id}+\frac{1}{2} \xi_{\sigma}^{t} \circ \xi_{\sigma}\right)} d v_{g}
$$

In view of our applications, we also consider $\sigma$ as a map $\sigma:(M, \tilde{g}) \rightarrow\left(\Lambda_{q}(M), g^{S}\right)$, where $\tilde{g}$ is an arbitrary metric on $M$, which may be different from $g$. Denote by $\mathcal{E}_{\tilde{g}}(\sigma)$ the energy of $\sigma$ as a map between these Riemannian manifolds. Let $L_{\sigma, \tilde{g}}$ and $Q_{\tilde{g}}$ be the automorphism fields determined, respectively by

$$
\left(\sigma^{*} g^{S}\right)(X, Y)=\tilde{g}\left(L_{\sigma, \tilde{g}} X, Y\right), \quad \tilde{g}(X, Y)=g\left(Q_{\tilde{g}} X, Y\right) .
$$

Then $L_{\sigma}=Q_{\tilde{g}} \circ L_{\sigma, \tilde{g}}$ and the energy of $\sigma$ can be written as

$$
\mathcal{E}_{\tilde{g}}(\sigma)=\frac{1}{2} \int_{M} \operatorname{trace}\left(Q_{\tilde{g}}^{-1} \circ L_{\sigma}\right) \sqrt{\operatorname{det} Q_{\tilde{g}}} d v_{g} .
$$


In particular, for $\tilde{g}=g, \mathcal{E}(\sigma)=\mathcal{E}_{\tilde{g}}(\sigma)$ is the energy of the section $\sigma$, and, for $\tilde{g}=\sigma^{*} g^{S}$, we have $Q_{\tilde{g}}=L_{\sigma}$ and so

$$
\operatorname{Vol}(\sigma)=\frac{2}{n} \mathcal{E}_{\left(\sigma^{*} g^{S}\right)}(\sigma)
$$

Denote by $\tau_{\tilde{g}}(\sigma)$ the tension field of $\sigma$, i.e., the vector field along $\sigma$ given locally by

$$
\tau_{\tilde{g}}(\sigma)=\nabla_{\tilde{E}_{i}}^{S} \sigma_{*} \tilde{E}_{i}-\sigma_{*} \tilde{\nabla}_{\tilde{E}_{i}} \tilde{E}_{i}
$$

where $\tilde{\nabla}$ and $\nabla^{S}$ denote the Levi-Civita connections of $\tilde{g}$ and $g^{S}$, respectively, ( $\nabla^{S}$ is considered as the connection of $g^{S}$ on the induced vector bundle $\sigma^{*} T \Lambda_{q}(M)$ ) and $\left\{\tilde{E}_{i}\right\}_{i=1}^{n}$ is a local $\tilde{g}$-orthonormal frame. In [8], $\tau_{\tilde{g}}(\sigma)$ has been characterized as follows:

$$
\tau_{\tilde{g}}(\sigma)=\left(X_{\sigma, \tilde{g}}\right)^{\text {hor }} \circ \sigma+\left(\eta_{\sigma, \tilde{g}}\right)^{\text {vert }} \circ \sigma,
$$

where $\left(X_{\sigma, \tilde{g}}\right)^{\text {hor }}$ is the horizontal lift on $\Lambda_{q}(M)$ of $X_{\sigma, \tilde{g}}=\left(R_{\sigma, \tilde{g}}\right)^{\sharp}+\tau_{\tilde{g}}(\mathrm{Id}), R_{\sigma, \tilde{g}}$ is the one-form on $M$ defined locally by

$$
R_{\sigma, \tilde{g}}(X)=g\left(R_{X \tilde{E}_{i}} \sigma, \nabla_{\tilde{E}_{i}} \sigma\right)
$$

$\tau_{\tilde{g}}(\mathrm{Id})$ is the tension of Id $:(M, \tilde{g}) \rightarrow(M, g)$, and $\left(\eta_{\sigma, \tilde{g}}\right)^{\text {vert }}$ is the vertical lift of $\eta_{\sigma, \tilde{g}} \in \Gamma^{\infty}\left(\Lambda_{q}(M)\right)$, defined on the domain of $\left\{\tilde{E}_{i}\right\}$ by

$$
\eta_{\sigma, \tilde{g}}=\nabla_{\tau_{\tilde{g}}(\mathrm{Id})} \sigma+\left(\nabla^{2} \sigma\right)_{\tilde{E}_{i} \tilde{E}_{i}}
$$

Here $R_{X Y} \sigma=\nabla_{[X, Y]} \sigma-\nabla_{X} \nabla_{Y} \sigma+\nabla_{Y} \nabla_{X} \sigma$ and $\left(\nabla^{2} \sigma\right)_{X Y}=\nabla_{X} \nabla_{Y} \sigma-\nabla_{\left(\nabla_{X} Y\right)} \sigma$.

Hence, the map $\sigma:(M, \tilde{g}) \rightarrow\left(\Lambda_{q}(M), g^{S}\right)$ is harmonic if and only if $X_{\sigma, \tilde{g}}=0$ and $\eta_{\sigma, \tilde{g}}=0$. For $\tilde{g}=g$, putting $R_{\sigma}=R_{\sigma, g}, \sigma$ is harmonic if and only if $R_{\sigma}=0$ and $\nabla^{*} \nabla \sigma=0$, where $\nabla^{*} \nabla \sigma=-\left(\nabla^{2} \sigma\right)_{E_{i} E_{i}}$ is the connection Laplacian (or rough Laplacian) [17], $\left\{E_{i}\right\}_{i=1}^{n}$ being a local $g$-orthonormal frame. Because for compact $M$ the connection Laplacian of $\sigma$ vanishes if and only if $\sigma$ is parallel (see page 154 of [17]), the harmonicity condition for $\sigma$ is equivalent to $\sigma$ being parallel.

Next, let $K_{\sigma, \tilde{g}}$ be the $C^{\infty}(M)$-linear map $K_{\sigma, \tilde{g}}: \mathfrak{X}(M) \rightarrow \Gamma^{\infty}\left(\Lambda_{q}(M)\right)$ given by

$$
K_{\sigma, \tilde{g}}=\sqrt{\operatorname{det} Q_{\tilde{g}}}(\nabla \sigma) \circ Q_{\tilde{g}}^{-1} .
$$

Then, in [8] it is proved that

$$
\nabla^{*} K_{\sigma, \tilde{g}}=-\sqrt{\operatorname{det} Q_{\tilde{g}}} \eta_{\sigma, \tilde{g}}
$$

where $\nabla^{*} K_{\sigma, \tilde{g}}$ is defined locally by $\nabla^{*} K_{\sigma, \tilde{g}}=-\left(\nabla_{E_{i}} K_{\sigma, \tilde{g}}\right) E_{i}$. Since for the case $\tilde{g}=\sigma^{*} g^{S}$, the condition $\eta_{\sigma, \tilde{g}}=0$ implies that $X_{\sigma, \tilde{g}}=0$, [8], one obtains from (3.7) and (3.9) that the immersion $\sigma: M \rightarrow\left(\Lambda_{q}(M), g^{S}\right)$ is minimal if and only if $\nabla^{*} K_{\sigma}=0$, where $K_{\sigma}$ is given by

$$
K_{\sigma}=\sqrt{\operatorname{det} L_{\sigma}}(\nabla \sigma) \circ L_{\sigma}^{-1} .
$$


Denote by $S_{\sigma(x)}^{0}$ the subspace of $\Lambda^{q}\left(T_{x} M\right)$ generated by $\sigma(x)$, by $S_{\sigma(x)}^{1}$ to the tangent space $T_{\sigma(x)}{ }^{\Sigma_{q}}\left(T_{x} M\right)$, generated by $\sigma_{j}^{a}, a=1, \ldots, q, j=1, \ldots, n-q$, and by $S_{\sigma(x)}^{2}$ the subspace generated by

$$
\left\{\sigma_{j k}^{a b}(x) \mid 1 \leq a<b \leq q ; 1 \leq i<j \leq n-q\right\}
$$

where $\sigma_{j k}^{a b}(x)=(-1)^{a+b} e_{q+j} \wedge e_{q+k} \wedge e_{1} \wedge \cdots \wedge \hat{e}_{a} \wedge \cdots \wedge \hat{e}_{b} \wedge \cdots \wedge e_{q}$. Then, using (3.3), for every $\sigma \in \Gamma^{\infty}\left(\Sigma_{q}(M)\right)$ we have

$$
\left(\nabla^{*} K_{\sigma, \tilde{g}}\right)(x) \in S_{\sigma(x)}^{0} \oplus S_{\sigma(x)}^{1} \oplus S_{\sigma(x)}^{2} .
$$

Since the harmonicity of $\sigma$ viewed as map into $\Sigma_{q}^{\text {or }}(M)$ is determined by the vanishing of the projection onto $T_{\sigma} \Sigma_{q}^{\text {or }}\left(T_{x} M\right)$ of the tension, the map $\sigma:(M, \tilde{g}) \rightarrow$ $\left(G_{q}^{\text {or }}(M), g^{K}\right)$ is harmonic if and only if $X_{\sigma, \tilde{g}}=0$ and $g\left(\nabla^{*} K_{\sigma, \tilde{g}}, \sigma_{j}^{a}\right)=0$. In particular, $\sigma:(M, g) \rightarrow\left(G_{q}^{\text {or }}(M), g^{K}\right)$ is harmonic if and only if $R_{\sigma}=0$ and $g\left(\nabla^{*} \nabla \sigma, \sigma_{j}^{a}\right)=0$. A critical point $\sigma$ of the energy functional $\mathcal{E}_{\tilde{g}}$ restricted to $\Gamma^{\infty}\left(G_{q}^{\text {or }}(M)\right)$ is called a $\tilde{g}$-harmonic distribution (or simply harmonic distribution if $\tilde{g}=g$ ); it is characterized by the vanishing of the vertical component of $\tau_{\tilde{g}}(\sigma): g\left(\nabla^{*} K_{\sigma, \tilde{g}}, \sigma_{j}^{a}\right)=0$.

When the immersion $\sigma: M \rightarrow\left(G_{q}^{\text {or }}(M), g^{K}\right)$ is minimal, $\sigma$ is called a minimal distribution. Then the minimality condition for a distribution $\sigma$ is determined by $g\left(\nabla^{*} K_{\sigma}, \sigma_{j}^{a}\right)=0$.

For general Riemannian manifolds $(M, g)$, not necessarily closed and oriented, we extend the above definitions for distributions satisfying such conditions.

Next, given a $(1,2)$-tensor field $\psi$ on $M$, we denote by $d^{*} \psi$ its coderivative, which is defined by

$$
\left(d^{*} \psi\right)_{x}(X)=-\left(\nabla_{e_{i}} \psi\right)_{e_{i}} X
$$

where $\left\{e_{1}, \ldots, e_{n}\right\}$ is any orthonormal basis of $T_{x} M$.

Theorem 3.4. Let $\sigma \in \Gamma^{\infty}\left(G_{q}^{\mathrm{or}}(M)\right)$ be an oriented $q$-dimensional distribution on a Riemannian manifold $(M, g)$ with intrinsic torsion $\xi$. Then, for any metric $\tilde{g}$, we have:

(i) the map $\sigma:(M, \tilde{g}) \rightarrow\left(G_{q}^{\text {or }}(M), g^{K}\right)$ is harmonic if and only if the following conditions are satisfied:

(a) $R_{\sigma, \tilde{g}}(X)+g\left(\tau_{\tilde{g}}(\mathrm{Id}), X\right)=0$, for all $X \in \mathfrak{X}(M)$, where $R_{\sigma, \tilde{g}}$ is expressed locally as $R_{\sigma, \tilde{g}}(X)=\frac{1}{2} g\left(R_{X \tilde{E}_{i}}, \xi_{\tilde{E}_{i}}\right)$, and

(b) $d^{*} \kappa_{\sigma, \tilde{g}}\left(\mathcal{V}_{\sigma}\right) \subset \mathcal{V}_{\sigma}\left(\right.$ or equivalently, $\left.d^{*} \kappa_{\sigma, \tilde{g}}\left(\mathcal{H}_{\sigma}\right) \subset \mathcal{H}_{\sigma}\right)$, where

$$
\kappa_{\sigma, \tilde{g}}=\sqrt{\operatorname{det} Q_{\tilde{g}}} \xi \circ Q_{\tilde{g}}^{-1} .
$$

In particular, the map $\sigma:(M, g) \rightarrow\left(G_{q}^{\mathrm{or}}(M), g^{K}\right)$ is harmonic if and only if

$$
d^{*} \xi=0 \quad \text { and } \quad R_{\sigma}=0
$$


(ii) the condition (b) characterizes $\tilde{g}$-harmonic distributions, and $d^{*} \xi=0$ characterizes harmonic distributions;

(iii) the immersion $\sigma: M \rightarrow\left(G_{q}^{\mathrm{or}}(M), g^{K}\right)$ is minimal if and only if

$$
d^{*} \kappa_{\sigma}\left(\mathcal{V}_{\sigma}\right) \subset \mathcal{V}_{\sigma} \quad\left(\text { or equivalently, } d^{*} \kappa_{\sigma}\left(\mathcal{H}_{\sigma}\right) \subset \mathcal{H}_{\sigma}\right)
$$

where $\kappa_{\sigma}=\sqrt{\operatorname{det} L_{\sigma}} \xi \circ L_{\sigma}^{-1}$ and $L_{\sigma}=\mathrm{Id}+\xi^{t} \circ \xi$.

Remark 3.5. Because the projection $p_{r}: G_{q}^{\text {or }}(M) \rightarrow G_{q}(M)$ is a local isometry with respect to the corresponding Kaluza-Klein metrics, the conditions which a (unoriented) distribution $\sigma:(M, \tilde{g}) \rightarrow\left(G_{q}(M), g^{K}\right)$ must satisfy in order that $\sigma$ be a harmonic map, or a $\tilde{g}$-harmonic distribution, or the immersion $\sigma: M \rightarrow$ $\left(G_{q}(M), g^{K}\right)$ be minimal, are, respectively, the conditions (i), (ii) or (iii) of Theorem 3.4 for oriented distributions. The condition that $\sigma$ be a harmonic distribution is also equivalent to $\left[P_{\sigma}, \nabla^{*} \nabla P_{\sigma}\right]=0$, where $[\cdot, \cdot]$ denotes the commutator bracket of endomorphisms (see [28]).

For the proof of Theorem 3.4, we shall need the following lemmas.

Lemma 3.6. Let $\sigma$ be a q-dimensional oriented distribution on a Riemannian manifold $(M, g)$ and let $A$ be a $(1,1)$-tensor field on $M$. We have:

(i) $d^{*}(\xi \circ A) \in \Gamma^{\infty}(\mathfrak{s o}(M))$ and, if $\left[\xi_{E_{i}}, \xi_{A E_{i}}\right]=0$, then $d^{*}(\xi \circ A) \in \Gamma^{\infty}\left(\mathfrak{m}_{\sigma}\right)$;

(ii) $g\left(\left(d^{*}(\xi \circ A) E_{a}, E_{q+j}\right)=g\left(\nabla^{*}(\nabla \sigma \circ A), \sigma_{j}^{a}\right)\right.$.

Proof. Because $\xi \in \Gamma^{\infty}\left(\mathfrak{m}_{\sigma}\right)$, we obtain $d^{*}(\xi \circ A) \in \Gamma^{\infty}(\mathfrak{s o}(M))$. For $U, V \in \mathcal{V}_{\sigma}$, using (2.2), one gets

$$
\begin{aligned}
g\left(d^{*}(\xi \circ A) U, V\right) & =g\left(\xi_{A E_{i}} \nabla_{E_{i}} U, V\right)-g\left(\nabla_{E_{i}} \xi_{A E_{i}} U, V\right) \\
& =g\left(\xi_{A E_{i}} U, \xi_{E_{i}} V\right)-g\left(\xi_{E_{i}} U, \xi_{A E_{i}} V\right)=-g\left(\left[\xi_{E_{i}}, \xi_{A E_{i}}\right] U, V\right) .
\end{aligned}
$$

In the same way, $g\left(d^{*}(\xi \circ A) X, Y\right)=-g\left(\left[\xi_{E_{i}}, \xi_{A E_{i}}\right] X, Y\right)$, for all $X, Y \in \mathcal{H}_{\sigma}$. Hence, if $\left[\xi_{E_{i}}, \xi_{A E_{i}}\right]=0$, then $d^{*}(\xi \circ A) \mathcal{V}_{\sigma} \subset \mathcal{H}_{\sigma}$ and $d^{*}(\xi \circ A) \mathcal{H}_{\sigma} \subset \mathcal{V}_{\sigma}$, which proves (i). Next, we show (ii). From (3.3) we get

$$
\nabla_{X} \nabla_{Y} \sigma=X g\left(\xi_{Y} E_{a}, E_{q+j}\right) \sigma_{j}^{a}+g\left(\xi_{Y} E_{a}, E_{q+j}\right) \nabla_{X} \sigma_{j}^{a}
$$

and the projection of $\nabla_{X} \sigma_{j}^{a}$ onto $S_{\sigma}^{1}$ is given by

$$
g\left(\nabla_{X} E_{q+j}, E_{q+k}\right) \sigma_{k}^{a}-g\left(\nabla_{X} E_{b}, E_{a}\right) \sigma_{j}^{b} .
$$

Then, we have

$$
\begin{aligned}
g\left(\nabla_{X} \nabla_{Y} \sigma, \sigma_{j}^{a}\right)= & g\left(\nabla_{X} \xi_{Y} E_{a}, E_{q+j}\right)+g\left(\xi_{Y} E_{a}, \nabla_{X} E_{q+j}\right) \\
& -g\left(\xi_{Y} E_{a}, E_{q+k}\right) g\left(\nabla_{X} E_{q+j}, E_{q+k}\right)+g\left(\nabla_{X} E_{a}, E_{b}\right) g\left(\xi_{Y} E_{q+j}, E_{b}\right) \\
= & g\left(\nabla_{X} \xi_{Y} E_{a}, E_{q+j}\right)+g\left(\nabla_{X} E_{a}, \xi_{Y} E_{q+j}\right) .
\end{aligned}
$$


Hence, one gets $g\left(\nabla_{X} \nabla_{Y} \sigma, \sigma_{j}^{a}\right)=g\left(\left(\nabla_{X} \xi_{Y}\right) E_{a}, E_{q+j}\right)$. Then, using again (3.3),

$$
g\left(\left(\nabla_{X}(\nabla \sigma \circ A)\right) Y, \sigma_{j}^{a}\right)=g\left(\left(\nabla_{X}(\xi \circ A)\right)_{Y} E_{a}, E_{q+j}\right)
$$

and this proves (ii).

Lemma 3.7. We have:

(i) $g\left(R_{X Y} E_{a}, E_{q+j}\right)=g\left(R_{X Y} \sigma, \sigma_{j}^{a}\right)$;

(ii) $R_{\sigma, \tilde{g}}(X)=g\left(R_{X \tilde{E}_{i}} E_{a}, \xi_{\tilde{E}_{i}} E_{a}\right)=g\left(R_{X \tilde{E}_{i}} E_{q+j}, \xi_{\tilde{E}_{i}} E_{q+j}\right)=\frac{1}{2} g\left(R_{X \tilde{E}_{i}}, \xi_{\tilde{E}_{i}}\right)$.

Proof. A straightforward calculation, using (2.2), gives

$$
g\left(R_{X Y} E_{a}, E_{q+j}\right)=g\left(\left(\nabla_{Y} \xi\right)_{X} E_{a}-\left(\nabla_{X} \xi\right)_{Y} E_{a}, E_{q+j}\right) .
$$

Because $g\left(R_{X Y} \sigma, \sigma_{j}^{a}\right)=g\left(\nabla_{Y X}^{2} \sigma-\nabla_{X Y}^{2} \sigma, \sigma_{j}^{a}\right)$, (i) is obtained from (3.12). From this, and using (3.3), we get $R_{\sigma, \tilde{g}}(X)=g\left(R_{X \tilde{E}_{i}} E_{a}, E_{q+j}\right) g\left(\xi_{\tilde{E}_{i}} E_{a}, E_{q+j}\right)$. Then (ii) is also proved.

Proof of Theorem 3.4. The map $\sigma:(M, \tilde{g}) \rightarrow\left(G_{q}^{\text {or }}(M), g^{K}\right)$ is harmonic if and only if $X_{\sigma, \tilde{g}}=0$ and $g\left(\nabla^{*} K_{\sigma, \tilde{g}}, \sigma_{j}^{a}\right)=0$. From the definition of $X_{\sigma, \tilde{g}}$, it vanishes if and only if $R_{\sigma, \tilde{g}}(X)+g\left(\tau_{\tilde{g}}(\mathrm{Id}), X\right)=0$, for all $X \in \mathfrak{X}(M)$, where, using Lemma 3.7 (ii), $R_{\sigma, \tilde{g}}$ is expressed in terms of the intrinsic torsion as $R_{\sigma, \tilde{g}}(X)=\frac{1}{2} g\left(R_{X \tilde{E}_{i}}, \xi_{\tilde{E}_{i}}\right)$.

On the other hand, from Lemma 3.6, we obtain

$$
\begin{aligned}
g\left(\nabla^{*} K_{\sigma, \tilde{g}}, \sigma_{j}^{a}\right) & =g\left(\nabla^{*}\left(\nabla \sigma \circ\left(\sqrt{\operatorname{det} Q_{\tilde{g}}} Q_{\tilde{g}}^{-1}\right)\right), \sigma_{j}^{a}\right) \\
& =g\left(\left(d^{*} \kappa_{\sigma, \tilde{g}}\right) E_{a}, E_{q+j}\right)=-g\left(E_{a},\left(d^{*} \kappa_{\sigma, \tilde{g}}\right) E_{q+j}\right) .
\end{aligned}
$$

Hence, the vanishing of $g\left(\nabla^{*} K_{\sigma, \tilde{g}}, \sigma_{j}^{a}\right)$ is equivalent to each of the conditions for $d^{*} \kappa_{\sigma, \tilde{g}}$ given in (b), and we have proved (i). Now, the rest of the proof is a direct consequence of this statement.

\section{Transversally symmetric invariant foliations}

Let $(M, g)$ be a connected homogeneous Riemannian manifold. Then $(M, g)$ can be expressed as a coset space $G / K$, where $G$ is a Lie group, which assumed to be connected, acting transitively and effectively on $M ; K$ is the isotropy subgroup of $G$ at some point $o \in M$, the origin of $G / K$; and $g$ is a $G$-invariant Riemannian metric. We say that $g$ is a homogeneous Riemannian metric of $M$. Moreover, we can assume that $G / K$ is a reductive homogeneous space, i.e., there is an $\operatorname{Ad}(K)$ invariant subspace $\mathfrak{m}$ of the Lie algebra $\mathfrak{g}$ of $G$ such that $\mathfrak{g}=\mathfrak{k} \oplus \mathfrak{m}, \mathfrak{k}$ being the Lie algebra of $K$. Then, under the identification $\mathfrak{m} \cong T_{o} M, g$ corresponds with an $\operatorname{Ad}(K)$-invariant inner product $\langle\cdot, \cdot\rangle$ on $\mathfrak{m}$. When there exists an $\operatorname{Ad}(G)$-invariant inner product on $\mathfrak{g}$, which we also denote by $\langle\cdot, \cdot\rangle$, whose restriction to $\mathfrak{m}=\mathfrak{k}^{\perp}$ is $\langle\cdot, \cdot\rangle$, the space $(M=G / K, g)$ is called normal homogeneous. 
Let $\tilde{T}$ denote the torsion tensor and $\tilde{R}$ the curvature tensor of the canonical connection $\tilde{\nabla}$ of $(M, g)$ adapted to the reductive decomposition $\mathfrak{g}=\mathfrak{k} \oplus \mathfrak{m}$ (see [16], II, page 190). At the origin, they are given by

$$
\tilde{T}_{o}(X, Y)=-[X, Y]_{\mathfrak{m}} \quad, \quad \tilde{R}_{o}(X, Y)=\operatorname{ad}_{[X, Y]_{\mathfrak{k}}},
$$

where $[X, Y]_{\mathfrak{m}}$ and $[X, Y]_{\mathfrak{k}}$ denote respectively the $\mathfrak{m}$ - and $\mathfrak{k}$-component of $[X, Y]$ for all $X, Y \in \mathfrak{m}$. For each $X \in \mathfrak{m}$, consider $X^{+}$, the $G$-invariant vector field defined on a small neighborhood of $o$ in $M=G / K$ such that $X_{o}^{+}=X$, [18]. Then $\left[X^{+}, Y^{+}\right]_{o}=[X, Y]_{\mathfrak{m}}$ and the $G$-invariant connection $\tilde{\nabla}$ is determined by $\tilde{\nabla}_{X} Y^{+}=0$. Using the Koszul formula, the Levi-Civita connection $\nabla$ is given by

$$
2\left\langle\nabla_{X} Y^{+}, Z\right\rangle=-\left\langle X,[Y, Z]_{\mathfrak{m}}\right\rangle-\left\langle Y,[X, Z]_{\mathfrak{m}}\right\rangle+\left\langle Z,[X, Y]_{\mathfrak{m}}\right\rangle,
$$

for $X, Y, Z \in \mathfrak{m}$. Let $\mathfrak{U}: \mathfrak{m} \times \mathfrak{m} \rightarrow \mathfrak{m}$ be the symmetric bilinear mapping defined by

$$
2\langle\mathfrak{U}(X, Y), Z\rangle=\left\langle[Z, X]_{\mathfrak{m}}, Y\right\rangle+\left\langle[Z, Y]_{\mathfrak{m}}, X\right\rangle .
$$

Then the homogeneous structure $S=\nabla-\tilde{\nabla}$ associated to $\mathfrak{g}=\mathfrak{k} \oplus \mathfrak{m}[24]$ is given by

$$
S_{X} Y=\nabla_{X} Y^{+}=\frac{1}{2}[X, Y]_{\mathfrak{m}}+\mathfrak{U}(X, Y), \text { for all } X, Y \in \mathfrak{m} .
$$

Note that $\left\langle S_{X} Y, Z\right\rangle+\left\langle Y, S_{X} Z\right\rangle=0$. When $\mathfrak{U}=0,(M=G / K, g)$ is said to be $n a$ turally reductive. Using (4.1), we have the following expression for the Riemannian curvature $R$ of $\nabla$ at the origin:

$$
R_{X Y}=\operatorname{ad}_{[X, Y]_{\mathfrak{k}}}+S_{[X, Y]_{\mathfrak{m}}}-\left[S_{X}, S_{Y}\right] .
$$

A $q$-dimensional distribution $\sigma$ on $(M=G / K, g)$ is said to be $G$-invariant if $a_{* x} \sigma(x)=\sigma(a \cdot x)$, for all $a \in G$ and $x \in M$, where $a_{* x}$ denotes the differential map of $a$ at $x$. Since the linear isotropy representation, i.e., the differential of the action of $K$ on $T_{o} M$, corresponds, under the identification $\mathfrak{m} \cong T_{o} M$, with the adjoint representation $\operatorname{Ad}(K)$ of $K$ on $\mathfrak{m}$, there exists a bijection between $\operatorname{Ad}(K)$-invariant subspaces $\mathfrak{p}=\sigma(o) \subset \mathfrak{m}$ and $G$-invariant distributions on $(M, g)$. The $\langle\cdot, \cdot\rangle$-orthogonal complement $\mathfrak{n}$ of $\mathfrak{p}$ in $\mathfrak{m}$ is also $\operatorname{Ad}(K)$-invariant and the pair $(\mathfrak{p}, \mathfrak{n})$ determines an invariant orthogonal distribution $\left(\mathcal{V}_{\sigma}, \mathcal{H}_{\sigma}\right)$. Hence, the intrinsic torsion is invariant and the existence of invariant distributions implies that $G / K$ must be isotropy-reducible. Moreover, if $\left\{E_{a} ; E_{q+j}\right\}$ is an adapted orthonormal basis of $\mathfrak{m}=\mathfrak{p} \oplus \mathfrak{n}$, then $\left\{E_{a}^{+} ; E_{q+j}^{+}\right\}$is a local orthonormal frame around the origin adapted to $\left(\mathcal{V}_{\sigma}, \mathcal{H}_{\sigma}\right)$.

From (2.2) and (4.4), we have:

Lemma 4.1. Let $(M=G / K, g)$ be a homogeneous Riemannian manifold with adapted reductive decomposition $\mathfrak{g}=\mathfrak{k} \oplus \mathfrak{m}$ and let $\sigma$ be a $q$-dimensional invariant distribution determined by the pair $(\mathfrak{p}, \mathfrak{n})$. Then the intrinsic torsion $\xi$ at the origin of $\sigma$ is given by

$$
\begin{array}{ll}
\xi_{U} V=\left(S_{U} V\right)_{\mathfrak{n}}, & \xi_{U} X=\left(S_{U} X\right)_{\mathfrak{p}}, \\
\xi_{X} U=\left(S_{X} U\right)_{\mathfrak{n}}, & \xi_{X} Y=\left(S_{X} Y\right)_{\mathfrak{p}},
\end{array}
$$

for all $U, V \in \mathfrak{p}$ and $X, Y \in \mathfrak{n}$. 
Hence, $\sigma$ is

(i) totally geodesic if and only if $\mathfrak{U}(\mathfrak{p}, \mathfrak{p}) \subset \mathfrak{p}$;

(ii) integrable if and only if $[\mathfrak{p}, \mathfrak{p}]_{\mathfrak{m}} \subset \mathfrak{p}$. Moreover, the foliation is Riemannian if and only if $\mathfrak{U}(\mathfrak{n}, \mathfrak{n}) \subset \mathfrak{n}$.

Then any G-invariant Riemannian AP-structure on a naturally reductive homogeneous space is totally geodesic, i.e., both distributions $\mathcal{V}$ and $\mathcal{H}$ are totally geodesic. If a $G$-invariant distribution is integrable, the corresponding foliation $\mathcal{F}$ is a $G$-invariant foliation, meaning $a \cdot \mathcal{L}_{x}=\mathcal{L}_{a \cdot x}$, for all $a \in G$ and $x \in M$, where $\mathcal{L}_{x}$ denotes the leaf through $x$, and conversely, and, by Lemma 4.1 (ii), $\mathfrak{l}=\mathfrak{k} \oplus \mathfrak{p}$ is a Lie subalgebra of $\mathfrak{g}$. We shall denote by $L$ the connected Lie subgroup of $G$ with Lie algebra $\mathfrak{l}$.

A foliation $\mathcal{F}$ on a differentiable manifold $M$ is called transversally symmetric if there exists a symmetric pair $(G, L)$ such that its transversal geometry is locally modeled on $G / L$. This means that $\mathcal{F}$ can be defined by a family of local submersions $f_{i}: \mathcal{U}_{i} \rightarrow G / L$ such that $\left\{\mathcal{U}_{i}\right\}$ is an open covering of $M$ and $f_{j}=a_{j i} \cdot f_{i}$ with $a_{j i} \in G$.

In the context of $G$-invariant foliations, we say that a $G$-invariant foliation $\mathcal{F}$ determined by an $\operatorname{Ad}(K)$-invariant subspace $\mathfrak{p} \subset \mathfrak{m}$ is transversally symmetric if $(\mathfrak{g}, \mathfrak{s})$ is an orthogonal symmetric Lie algebra, where $\mathfrak{s}$ is the involutive automorphism of $\mathfrak{g}$ whose \pm 1 -eigenspaces are $\mathfrak{l}$ and $\mathfrak{n}$, respectively. Then the brackets satisfy

$$
[\mathfrak{p}, \mathfrak{n}] \subset \mathfrak{n}, \quad[\mathfrak{n}, \mathfrak{n}]_{\mathfrak{m}} \subset \mathfrak{p}
$$

Since the connected Lie subgroup $\tilde{L}$ of the universal covering $\tilde{G}$ of $G$ with Lie algebra $\mathfrak{l}$ is closed, $(\tilde{G}, \tilde{L})$ is a symmetric pair associated with $(\mathfrak{g}, \mathfrak{s})$ (see Proposition 3.6, Chapter IV, in [15]). Hence, if $\mathcal{F}$ on $M$ is a transversally symmetric invariant foliation then it is in fact locally modeled on the simply connected symmetric space $\tilde{G} / \tilde{L}$.

Another homogeneous Riemannian metric $\tilde{g}$ on $M$ is said to be adapted to the AP-structure $(\mathcal{V}, \mathcal{H})$, if the distributions $\mathcal{V}$ and $\mathcal{H}$ are $\tilde{g}$-orthogonal. It is clear that if an invariant foliation is transversally symmetric, it is also transversally symmetric for any adapted homogeneous Riemannian metric.

Using (4.7) and substituting in (4.3), one gets $\mathfrak{U}(\mathfrak{p}, \mathfrak{p}) \subset \mathfrak{p}$. Hence, transversally symmetric $G$-invariant foliations are totally geodesic, but not necessarily Riemannian foliations. In the next section we shall give examples of non-Riemannian transversally symmetric foliations. By Lemma 4.1 (ii), the foliation is Riemannian if and only if $\mathfrak{U}(\mathfrak{n}, \mathfrak{n})=0$, or equivalently, $S_{X} Y=\frac{1}{2}[X, Y]_{\mathfrak{m}}$, for all $X, Y \in \mathfrak{n}$.

Theorem 4.2. Let $\sigma$ be the distribution tangent to a transversally symmetric $G$-invariant foliation $\mathcal{F}$ of $(M=G / K, g)$. For any adapted homogeneous Riemannian metric $\tilde{g}$ on $G / K, \sigma$ is a $\tilde{g}$-harmonic distribution. In particular, $\sigma$ provides a minimal immersion into the Grassmannian bundle $\left(G_{q}(M), g^{K}\right)$. If moreover $\mathcal{F}$ is Riemannian, then $\sigma:(M, g) \rightarrow\left(G_{q}(M), g^{K}\right)$ is a harmonic map. 
Proof. Let $\mathfrak{p} \subset \mathfrak{m}$ the $A d(K)$-invariant subspace that determines the distribution $\sigma$. Then,

$$
S_{\mathfrak{p}} \mathfrak{p} \subset \mathfrak{p}, \quad S_{\mathfrak{p}} \mathfrak{n} \subset \mathfrak{n} .
$$

Moreover, by $(4.7), \mathfrak{U}(\mathfrak{n}, \mathfrak{n}) \subset \mathfrak{p}$ and $\mathfrak{U}(\mathfrak{p}, \mathfrak{n}) \subset \mathfrak{n}$, and this implies

$$
S_{\mathfrak{n}} \mathfrak{n} \subset \mathfrak{p}, \quad S_{\mathfrak{n}} \mathfrak{p} \subset \mathfrak{n} .
$$

Hence, with these hypotheses, one gets

$$
\xi_{\mathfrak{p}}=0, \quad \xi_{\mathfrak{n}}=S_{\mathfrak{n}} .
$$

Since $g$ and $\tilde{g}$ are $G$-invariant Riemannian metrics, the automorphism field $Q_{\tilde{g}}$ is also $G$-invariant and so it is determined by the $\operatorname{Ad}(K)$-invariant linear isomorphism $Q_{o}: \mathfrak{m} \rightarrow \mathfrak{m}$ defined by $\widetilde{\langle u, v\rangle}=\left\langle Q_{o} u, v\right\rangle$, where $\widetilde{\langle\cdot, \cdot\rangle}$ denotes the $\operatorname{Ad}(K)$-invariant inner product on $\mathfrak{m}$ corresponding to $\tilde{g}$. Taking into account that the subspaces $\mathfrak{p}$ and $\mathfrak{n}$ of $\mathfrak{m}$ must be orthogonal with respect to $\widetilde{\langle\cdot, \cdot\rangle}$, it follows that the decomposition $\mathfrak{m}=\mathfrak{p} \oplus \mathfrak{n}$ is $Q_{o}$-invariant. Hence, we can take the adapted $\langle\cdot, \cdot\rangle$-orthonormal basis $\left\{E_{a} ; E_{q+j}\right\}$ in $\mathfrak{m}$ as a basis of eigenvectors of $Q_{o}$. Then, applying (4.10) and also (4.8) and (4.9), we get

$$
\begin{aligned}
d^{*}\left(\xi \circ Q_{o}^{-1}\right) & =-\left(\nabla_{E_{i_{o}}} \xi_{Q_{o}^{-1} E_{i}}-\xi_{Q_{o}^{-1} \nabla_{E_{i}} E_{i}}-\xi_{Q_{o}^{-1} E_{i}} \nabla_{E_{i}}\right) \\
& =\left[\xi_{Q_{o}^{-1} E_{i}}, S_{E_{i}}\right]+\xi_{Q_{o}^{-1} S_{E_{i}} E_{i}}=\left[\xi_{Q_{o}^{-1} E_{q+j}}, S_{E_{q+j}}\right]=0 .
\end{aligned}
$$

Hence, using Theorem 3.4, $\sigma$ is a $\tilde{g}$-harmonic distribution. For $\tilde{g}=\sigma^{*} g^{S}$ we have $Q_{\tilde{g}}=L_{\sigma}$. Then $\tilde{g}$ is a homogeneous Riemannian metric on $M$ and, from (4.10), adapted to the AP-structure $\left(\mathcal{V}_{\sigma}, \mathcal{H}_{\sigma}\right)$. Thus Theorem 3.4 also implies that $\sigma$ is minimal.

From (4.5), and from (4.8) and (4.9), one gets $\left\langle R_{X Y} U, Z\right\rangle=0$, for all $U \in \mathfrak{p}$, and $X, Y, Z \in \mathfrak{n}$. Then, $\left(R_{\sigma}\right)_{o}(\mathfrak{n})=0$. On the other hand, for each $U \in \mathfrak{p}$, applying (4.10) and Lemma 3.7, together with the Riemannian condition for the foliation and (4.5), we obtain

$$
\begin{aligned}
\left(R_{\sigma}\right)_{o}(U) & =\left\langle R_{U E_{q+j}} E_{q+k}, \xi_{E_{q+j}} E_{q+k}\right\rangle \\
& =\left\langle S_{\left[U, E_{q+j}\right]} E_{q+k}+S_{E_{q+j}} S_{U} E_{q+k}, \xi_{E_{q+j}} E_{q+k}\right\rangle \\
& =\left\langle\xi_{E_{q+j}}\left(\left[U, E_{q+k}\right]+S_{U} E_{q+k}\right), \xi_{E_{q+j}} E_{q+k}\right\rangle \\
& =-\left\langle\left[U, E_{q+k}\right]+S_{U} E_{q+k}, \xi_{E_{q+j}}^{2} E_{q+k}\right\rangle .
\end{aligned}
$$

Because $\xi_{E_{q+j_{0}}}^{2}$ is a symmetric endomorphism with respect to $\langle\cdot, \cdot\rangle$ and preserves $\mathfrak{n}$, for each $j_{0} \in\{1, \ldots, n-q\}$, we can take $\left\{E_{q+1}, \ldots, E_{n}\right\}$ as a basis of eigenvectors of $\xi_{E_{q+j_{0}}}^{2}$. Denote by $\delta_{q+k}$ the corresponding eigenvalues. Then, using again that $\mathcal{F}$ is a Riemannian foliation, we obtain

$$
\left\langle\left[U, E_{q+k}\right]+S_{U} E_{q+k}, \xi_{E_{q+j_{0}}}^{2} E_{q+k}\right\rangle=\delta_{q+k}\left\langle\left[U, E_{q+k}\right]+S_{U} E_{q+k}, E_{q+k}\right\rangle=0 .
$$

Thus, $R_{\sigma}=0$ and, applying again Theorem 3.4, $\sigma$ is a harmonic map. 
Finally we consider transversally symmetric invariant foliations $\mathcal{F}$ on $(M=$ $G / K, g)$ such that the connected Lie subgroup $L$ of $G$ with Lie algebra $\mathfrak{l}=\mathfrak{k} \oplus \mathfrak{p}$ is closed. This is the case if the center of $\mathfrak{g}$ is $\{0\}$ (see the proof of Proposition 3.6 in Chapter IV of [15]), in particular, when $\mathfrak{g}$ is semisimple. With this hypothesis we have:

Proposition 4.3. The leaves of the foliation $\mathcal{F}$ of $M=G / K$ are the fibres of the homogeneous fibration

$$
F=L / K \rightarrow M=G / K \stackrel{\pi}{\rightarrow} N=G / L: g K \mapsto g L .
$$

The foliation $\mathcal{F}$ is moreover Riemannian if and only if the restriction $\langle\cdot, \cdot\rangle_{\mathfrak{n}}$ of $\langle\cdot, \cdot\rangle$ to $\mathfrak{n}$ is $\operatorname{Ad}(L)$-invariant. Then $\pi:(M, g) \rightarrow\left(N, g_{N}\right)$ is a Riemannian submersion and $\left(N, g_{N}\right)$ is a locally Riemannian symmetric space, where $g_{N}$ is the G-invariant metric on $N$ induced by $\langle\cdot, \cdot\rangle_{\mathfrak{n}}$.

Proof. From the uniqueness of maximal integral submanifolds through a point, $L / K$ is the leaf through the origin $o \in M$. For another point $g K \in G / K$, we use the following property: $\pi^{-1}(a L)=a \cdot L / K$, for each $a \in G$.

The metric $\langle\cdot, \cdot\rangle_{\mathfrak{n}}$ is $\operatorname{Ad}(L)$-invariant if and only if $\langle[X, U], Y\rangle=\langle X,[U, Y]\rangle$, for all $X, Y \in \mathfrak{n}$ and $U \in \mathfrak{p}$. From (4.3), this is equivalent to the condition $\mathfrak{U}(\mathfrak{n}, \mathfrak{n}) \subset \mathfrak{n}$, i.e., the foliation is Riemannian. For the last part of the proposition we use Proposition 3.6 in Chapter IV of [15].

\section{Examples of transversally symmetric fibrations}

A homogeneous fibration $\pi: G / K \rightarrow G / L$ as in Proposition 4.3 is called a transversally symmetric fibration. Given a $G$-invariant metric $g$ on $M, \pi$ is said to be $g$-Riemannian (or Riemannian with respect to $g$ ) if the foliation determined by its fibres is Riemannian. Next, we give a useful method for obtaining many examples of Riemannian and non-Riemannian transversally symmetric fibrations.

Let $G$ be a connected compact Lie group and let $K$ and $L$ be connected closed subgroups of $G$ such that $K \subsetneq L \subsetneq G$. We consider the natural projection $\pi: M=$ $G / K \rightarrow N=G / L, g K \mapsto g L$. Then $\pi$ gives a homogeneous fibration with fibre type $F=L / K$. Let $B$ be an $\operatorname{Ad}(G)$-invariant inner product $B$ on the Lie algebra $\mathfrak{g}$ of $G$, and let $\mathfrak{m}, \mathfrak{p}$ and $\mathfrak{n}$ be subspaces of $\mathfrak{g}$ making $B$-orthogonal the following decompositions: $\mathfrak{g}=\mathfrak{k} \oplus \mathfrak{m}, \mathfrak{l}=\mathfrak{k} \oplus \mathfrak{p}$ and $\mathfrak{g}=\mathfrak{l} \oplus \mathfrak{n}$. Then $\mathfrak{m}=\mathfrak{p} \oplus \mathfrak{n}$ is also a $B$-orthogonal decomposition.

Lemma 5.1. The B-orthogonal decompositions $\mathfrak{l}=\mathfrak{k} \oplus \mathfrak{p}$ and $\mathfrak{g}=\mathfrak{l} \oplus \mathfrak{n}$ determine reductive decompositions for $F$ and $N$, respectively, and $\mathfrak{m}=\mathfrak{p} \oplus \mathfrak{n}$ is $\operatorname{Ad}(K)$ invariant.

Proof. Because $\mathfrak{p}$ is orthogonal to $\mathfrak{k}$, it is in fact a subspace of $\mathfrak{m}$ and, since $\mathfrak{l}$ is a Lie subalgebra, we get $[\mathfrak{k}, \mathfrak{p}]+[\mathfrak{p}, \mathfrak{p}] \subset \mathfrak{l}$. Then, $[\mathfrak{p}, \mathfrak{p}]_{\mathfrak{m}}=[\mathfrak{p}, \mathfrak{p}]_{\mathfrak{p}} \subset \mathfrak{p}$ and, taking into account that $\mathfrak{g}=\mathfrak{k} \oplus \mathfrak{m}$ is a reductive decomposition, it follows that $[\mathfrak{k}, \mathfrak{p}]$ is 
orthogonal to $\mathfrak{k}$ and so, $[\mathfrak{k}, \mathfrak{p}] \subset \mathfrak{p}$. Next, since $B$ is $\operatorname{Ad}(G)$-invariant, we obtain $B([\mathfrak{k}, \mathfrak{n}], \mathfrak{p})=-B([\mathfrak{k}, \mathfrak{p}], \mathfrak{n})=0$ and $B([\mathfrak{p}, \mathfrak{n}], \mathfrak{p})=-B([\mathfrak{p}, \mathfrak{p}], \mathfrak{n})=0$. This implies that $[\mathfrak{k}, \mathfrak{n}] \subset \mathfrak{n}$ and $[\mathfrak{p}, \mathfrak{n}] \subset \mathfrak{n}$, and consequently $[\mathfrak{l}, \mathfrak{n}] \subset \mathfrak{n}$. Now, using the inclusions $[\mathfrak{k}, \mathfrak{p}] \subset \mathfrak{p},[\mathfrak{k}, \mathfrak{n}] \subset \mathfrak{n}$ and $[\mathfrak{l}, \mathfrak{n}] \subset \mathfrak{n}$, together with the connectedness of $K$ and $L$, the result follows.

The isotropy group $K$ acts on $\mathfrak{m}$ by the adjoint map and induces a splitting

$$
\mathfrak{m}=\mathfrak{p} \oplus \mathfrak{n}=\left(\mathfrak{p}_{0} \oplus \mathfrak{p}_{1} \oplus \cdots \oplus \mathfrak{p}_{r}\right) \oplus\left(\mathfrak{n}_{0} \oplus \mathfrak{n}_{1} \oplus \cdots \oplus \mathfrak{n}_{s}\right),
$$

where $K$ acts trivially on $\mathfrak{m}_{0}=\mathfrak{p}_{0} \oplus \mathfrak{n}_{0}$ and irreducibly on $\mathfrak{p}_{i}$ and $\mathfrak{n}_{j}$, for $i=1, \ldots, r$ and $j=1, \ldots, s$. If the isotropy representations on $\mathfrak{p}_{i}$ and $\mathfrak{n}_{j}$ are inequivalent, any $G$-invariant Riemannian metric on $M=G / K$ is determined by an $\operatorname{Ad}(K)$-invariant inner product on $\mathfrak{m}$ of the form

$$
\langle\cdot, \cdot\rangle=\langle\cdot, \cdot\rangle_{0 \mid \mathfrak{m}_{0}}+\sum_{i=1}^{r} \lambda_{i} B_{\mid \mathfrak{p}_{i}}+\sum_{j=1}^{s} \mu_{j} B_{\mid \mathfrak{n}_{j}},
$$

$\langle\cdot, \cdot\rangle_{0}$ being an arbitrary inner product on $\mathfrak{m}_{0}$ and $\lambda_{i}, \mu_{j}>0, i=1, \ldots, r$, and $j=1, \ldots, s$. Hence, using Lemma 5.1 and taking into account that the subspace $\mathfrak{n} \subset \mathfrak{m}$ is $\operatorname{Ad}(L)$-invariant, the following proposition is proved.

Proposition 5.2. We have:

(i) If $\mathfrak{p}_{0}=\{0\}$ or $\mathfrak{n}_{0}=\{0\}$ then any homogeneous Riemannian metric on $M=$ $G / K$ is adapted to the $G$-invariant AP-structure generated by the pair $(\mathfrak{p}, \mathfrak{n})$.

(ii) For any homogeneous Riemannian metric adapted to $(\mathfrak{p}, \mathfrak{n})$, the fibration $\pi: M=G / K \rightarrow N=G / L$ is transversally symmetric if and only if $[\mathfrak{n}, \mathfrak{n}] \subset \mathfrak{l}$.

(iii) If the linear isotropy representation of $K$ on $\mathfrak{n}$ is irreducible, the fibration $\pi$ is $g$-Riemannian for any homogeneous Riemannian metric $g$ on $M$.

\subsection{Compact rank one normal homogeneous spaces}

Homogeneous spaces admitting a normal invariant metric of strictly positive curvature are called compact rank one normal homogeneous spaces (see, for example, [13] and [26], and the references therein). They are in fact compact and the positive normal homogeneous metric has rank one. In the simply connected case, a compact rank one normal homogeneous space is diffeomorphic to

(i) the sphere $S^{n}$ or one of the projective spaces $\mathbb{C} P^{m}, \mathbb{H} P^{m}$ and $\mathbb{C} a P^{2}$, or

(ii) one of the Berger spaces

$$
B^{7}=S p(2) / S U(2) \text { and } B^{13}=S U(5) /\left(\left(S p(2) \times S^{1}\right) /\{ \pm(\operatorname{Id}, 1)\}\right) \text {, or }
$$

(iii) the Wilking space $W^{7}=(S O(3) \times S U(3)) / U^{\bullet}(2)$. 


\begin{tabular}{llll}
\hline$G$ & $K$ & $\operatorname{dim} G / K$ & isotropy repr. \\
\hline (1) $S O(m+1)$ & $S O(m)$ & $m$ & irred. \\
(2) $S U(m+1)$ & $S U(m)$ & $2 m+1$ & $\mathfrak{m}=\mathfrak{p}_{0}^{1} \oplus \mathfrak{n}$ \\
(3) $U(m+1)$ & $U(m)$ & $2 m+1$ & $\mathfrak{m}=\mathfrak{p}_{0}^{1} \oplus \mathfrak{n}$ \\
(4) $S p(m+1)$ & $S p(m)$ & $4 m+3$ & $\mathfrak{m}=\mathfrak{p}_{0}^{3} \oplus \mathfrak{n}$ \\
(5) $S p(m+1) \times S p(1)$ & $S p(m) \times S p(1)$ & $4 m+3$ & $\mathfrak{m}=\mathfrak{p}_{1}^{3} \oplus \mathfrak{n}$ \\
(6) $S p(m+1) \times U(1)$ & $S p(m) \times U(1)$ & $4 m+3$ & $\mathfrak{m}=\mathfrak{p}_{0}^{2} \oplus \mathfrak{p} 1 \oplus \mathfrak{n}$ \\
(7) $S p i n(9)$ & $S p i n(7)$ & 15 & $\mathfrak{m}=\mathfrak{p} \oplus \mathfrak{n}$ \\
(8) $S p i n(7)$ & $G_{2}$ & 7 & irred. \\
(9) $G_{2}$ & $S U(3)$ & 6 & irred. \\
\hline
\end{tabular}

TABLE 1.

The (compact) Lie groups acting transitively on the sphere have been classified by D. Montgomery and H. Samelson, and A. Borel, see [19]. They are given, together with the isotropy representation, in Table 1. In Table 2, those corresponding to the exceptional cases are described. Here, the superscript represents the dimension of the subspace.

The unique projective space admitting an isotropy-reducible quotient expression is $\mathbb{C} P^{2 m+1}=S p(m+1) / S p(m) \times U(1)[19]$. Its isotropy representation determines the decomposition $\mathfrak{m}=\mathfrak{p}_{1}^{2} \oplus \mathfrak{n} \cong \mathfrak{p}_{1}^{2} \oplus \mathbb{H}^{m}$. All the homogeneous Riemannian metrics on these homogeneous spaces are obtained from inner products as in (5.1) and the following transversally symmetric homogeneous fibrations result:

(i) $S^{1} \rightarrow S^{2 m+1}=S U(m+1) / S U(m) \rightarrow \mathbb{C} P^{m}$,

(ii) $S^{2} \rightarrow \mathbb{C} P^{2 m+1}=S p(m+1) / S p(m) \times U(1) \rightarrow \mathbb{H} P^{m}$,

(iii) $S^{3} \rightarrow S^{4 m+3}=S p(m+1) / S p(m) \rightarrow \mathbb{H} P^{m}$,

(iv) $S^{7} \rightarrow S^{15}=\operatorname{Spin}(9) / \operatorname{Spin}(7) \rightarrow S^{8}$,

(v) $\mathbb{R} P^{5} \rightarrow B^{13} \rightarrow \mathbb{C} P^{4}$

(vi) $\mathbb{R} P^{3} \rightarrow W^{7} \rightarrow \mathbb{C} P^{2}$.

Because in all cases the subspace $\mathfrak{n} \subset \mathfrak{m}$ is irreducible under the linear isotropy action, it follows from Proposition 5.2 that the above six homogeneous fibrations are all Riemannian with respect to any invariant metric. The case (i) corresponds with the classical Hopf fibration. Note that the sets of $S U(m+1)$-invariant and of $U(m+1)$-invariant metrics coincide on $S^{2 m+1}$. The $S p(m+1) \times S p(1)$ invariant and $S p(m+1) \times U(1)$-invariant metrics on $S^{4 m+3}$, according to their corresponding isotropy representations, cases (5) and (6) in Table 1, are special classes of $S p(m+1)$-invariant metrics, as in case (4). Moreover, except for case (iii), any homogeneous Riemannian metric on the total space is binormal. 


\begin{tabular}{cccc}
\hline$G$ & $K$ & $G / K$ & isotropy repr. \\
\hline$S p(2)$ & $S U(2)$ & $B^{7}$ & irred. \\
$S U(5)$ & $\left(S p(2) \times S^{1}\right) / \pm(\mathrm{Id}, 1)$ & $B^{13}$ & $\mathfrak{m}=\mathfrak{p}^{5} \oplus \mathfrak{n}^{8}$ \\
$S O(3) \times S U(3)$ & $U^{\bullet}(2)$ & $W^{7}$ & $\mathfrak{m}=\mathfrak{p}^{3} \oplus \mathfrak{n}^{4}$ \\
\hline
\end{tabular}

TABLE 2 .

From Theorem 4.2, we have:

Theorem 5.3. For any homogeneous Riemannian metric on the total space of the homogeneous fibrations (i)-(vi), the distribution tangent to the fibres is minimal and determines a harmonic map into their Grassmannian bundles.

\subsection{Compact irreducible 3-symmetric spaces}

A Riemannian 3-symmetric space (see [14], [27] and also [9], [12]) is defined to be a triple $(M=G / K, \theta,\langle\cdot, \cdot\rangle)$ satisfying the following conditions:

(1) $G$ is a connected Lie group and $\theta$ is an automorphism of $G$ of order 3,

(2) $K$ is a closed subgroup of $G$ such that $G_{o}^{\theta} \subseteq K \subseteq G^{\theta}$, where $G^{\theta}=\{x \in G \mid$ $\theta(x)=x\}$ and $G_{o}^{\theta}$ denotes its identity component,

(3) $\langle\cdot, \cdot\rangle$ is an $\operatorname{Ad}(K)$ - and $\theta$-invariant inner product on $\mathfrak{m}=\operatorname{Ker} \phi$, where $\phi$ is the endomorphism of $\mathfrak{g}$ given by $\phi=1+\theta+\theta^{2}$.

If there exists $\theta$ satisfying (1) and (2), we shall say that $G / K$ is a 3 -symmetric coset space. The automorphism $J=\frac{1}{\sqrt{3}}\left(2 \theta_{\mid \mathfrak{m}}+\operatorname{Id}_{\mid \mathfrak{m}}\right)$ on $\mathfrak{m}$ is $\operatorname{Ad}(K)$-invariant and so it determines a $G$-invariant almost complex structure on $G / K$, known as the canonical almost complex structure. It makes the 3 -symmetric space a quasi-Kähler homogeneous manifold.

We will focus on compact irreducible Riemannian 3-symmetric spaces $(M=$ $G / K, \theta,\langle\cdot, \cdot\rangle)$ of type $A_{3}$, meaning that $G$ is a compact connected simple Lie group acting effectively and $\theta$ is an inner automorphism on the Lie algebra $\mathfrak{g}$ of $G$.

We need some general results about complex simple Lie algebras. See Chapter III in [15] for more details. Let $\mathfrak{g}_{\mathbb{C}}$ be a simple Lie algebra over $\mathbb{C}$ and let $\mathfrak{h}_{\mathbb{C}}$ be a Cartan subalgebra. Let $\Delta$ denote the set of nonzero roots of $\mathfrak{g}_{\mathbb{C}}$ with respect to $\mathfrak{h}_{\mathbb{C}}$ and let $\Pi=\left\{\alpha_{1}, \ldots, \alpha_{l}\right\}$ be a system of simple roots. For each $\alpha \in \Delta$, there exists a unique element $H_{\alpha} \in \mathfrak{h}_{\mathbb{C}}$ such that $C\left(H, H_{\alpha}\right)=\alpha(H)$, for all $H \in \mathfrak{h}_{\mathbb{C}}$, where $C$ is the Cartan-Killing form of $\mathfrak{g}_{\mathbb{C}}$. Moreover, we have $\mathfrak{h}_{\mathbb{C}}=\sum_{\alpha \in \Delta} \mathbb{C} H_{\alpha}$ and $C$ is strictly positive definite on $\mathfrak{h}_{\mathbb{R}}=\sum_{\alpha \in \Delta} \mathbb{R} H_{\alpha}$. We can choose root vectors $\left\{E_{\alpha}\right\}_{\alpha \in \Delta}$ such that $\mathfrak{g}_{\mathbb{C}}=\mathfrak{h}_{\mathbb{C}}+\sum_{\alpha \in \Delta} \mathbb{C} E_{\alpha}$ and, if $\alpha+\beta \neq 0, E_{\alpha}$ and $E_{\beta}$ are orthogonal under $C$ and $C\left(E_{\alpha}, E_{-\alpha}\right)=1$.

Denote by $\Delta^{+}$the set of positive roots of $\Delta$ with respect to some lexicographic order in $\Pi$. Then each $\alpha \in \Delta^{+}$may be written as $\alpha=\sum_{k=1}^{l} n_{k}(\alpha) \alpha_{k}$, where 


\begin{tabular}{clcc}
\hline Type & $\theta$ & $m_{i}$ & $\Pi(H)$ \\
\hline$A_{3} I$ & $\operatorname{Ad}_{\exp \frac{2 \pi \sqrt{-1}}{3} H_{i}}$ & 1 & $\left\{\alpha_{k} \in \Pi \mid k \neq i\right\}$ \\
$A_{3} I I$ & $\operatorname{Ad}_{\exp 2 \pi \sqrt{-1} \frac{\left(H_{i}+H_{j}\right)}{3}}$ & $m_{i}=m_{j}=1$ & $\left\{\alpha_{k} \in \Pi \mid k \neq i, k \neq j\right\}$ \\
$A_{3} I I I$ & $\operatorname{Ad}_{\exp \frac{4 \pi \sqrt{-1}}{3} H_{i}}$ & 2 & $\left\{\alpha_{k} \in \Pi \mid k \neq i\right\}$ \\
$A_{3} I V$ & $\operatorname{Ad}_{\exp 2 \pi \sqrt{-1} H_{i}}$ & 3 & $\left\{\alpha_{k} \in \Pi \mid k \neq i\right\} \cup\{-\mu\}$ \\
\hline
\end{tabular}

TABLE 3.

$n_{k}(\alpha) \in \mathbb{Z}, n_{k}(\alpha) \geq 0$, for all $k=1, \ldots, l$. The $\mathbb{R}$-linear subspace $\mathfrak{g}$ of $\mathfrak{g}_{\mathbb{C}}$ given by

$$
\mathfrak{g}=\mathfrak{h}+\sum_{\alpha \in \Delta^{+}}\left(\mathbb{R} U_{\alpha}^{0}+\mathbb{R} U_{\alpha}^{1}\right)
$$

is a compact real form of $\mathfrak{g}_{\mathbb{C}}$, where $\mathfrak{h}=\sum_{\alpha \in \Delta} \mathbb{R} \sqrt{-1} H_{\alpha}$ and $U_{\alpha}^{0}=E_{\alpha}-E_{-\alpha}=$ $-U_{-\alpha}^{0}$ and $U_{\alpha}^{1}=\sqrt{-1}\left(E_{\alpha}+E_{-\alpha}\right)=U_{-\alpha}^{1}$ (see Theorem 6.3 in Chapter III of [15]). Let $\mu=\sum_{i=1}^{l} m_{i} \alpha_{i}$ be the maximal root of $\Delta$ and consider $H_{i} \in \mathfrak{h}_{\mathbb{C}}, i=1, \ldots, l$, given by

$$
\alpha_{j}\left(H_{i}\right)=\frac{1}{m_{i}} \delta_{i j}, \quad i, j=1, \ldots, l .
$$

An inner automorphism $\theta$ of order 3 on $\mathfrak{g}_{\mathbb{C}}$ can be written, up to conjugation, as $\theta=\operatorname{Ad}_{\exp 2 \pi \sqrt{-1} H}$, where $H=\frac{1}{3} m_{i} H_{i}$ with $1 \leq m_{i} \leq 3$ or $H=\frac{1}{3}\left(H_{i}+H_{j}\right)$ with $m_{i}=m_{j}=1$. Then there are four classes for $\theta$ with corresponding simple root systems $\Pi(H)$ for $\mathfrak{g}_{\mathbb{C}}^{\theta}$, namely types $A_{3} I-A_{3} I V$, given in Table 3 . We have $\mathfrak{h} \subset \mathfrak{k}=\mathfrak{g}^{\theta}$ and

$$
\mathfrak{k}=\mathfrak{h}+\sum_{\alpha \in \Delta^{+}(H)}\left(\mathbb{R} U_{\alpha}^{0}+\mathbb{R} U_{\alpha}^{1}\right),
$$

where $\Delta^{+}(H)$ denotes the positive root system generated by $\Pi(H)$. Hence, each $\operatorname{Ad}(K)$-invariant inner product $\langle\cdot, \cdot\rangle$ on $\mathfrak{m}$ is also $\theta$-invariant and so, every 3 symmetric coset space $G / K$ with $\theta$ of type $A_{3}$ is a Riemannian 3-symmetric space for any $G$-invariant metric. Moreover, $\left\{U_{\alpha}^{0}, U_{\alpha}^{1} \mid \alpha \in \Delta^{+} \backslash \Delta^{+}(H)\right\}$ is an orthonormal basis for $\left(\mathfrak{m}, B_{\mid \mathfrak{m}}\right)$, where $B=-\frac{1}{2} C$.

Compact irreducible 3 -symmetric spaces of type $A_{3} I$ are Hermitian symmetric spaces. Together with those of type $A_{3} I V$, they are isotropy irreducible. So, in order to obtain homogeneous fibrations, we consider only irreducible 3 -symmetric spaces of types $A_{3} I I$ and $A_{3} I I I$.

If $M=G / K$ is of type $A_{3} I I$ then $m_{i}=m_{j}=1$, for some $i, j \in\{1, \ldots, l\}$, $i<j$. Let $\Delta_{p, q}=\left\{\alpha \in \Delta \mid n_{i}(\alpha)=p, n_{j}(\alpha)=q\right\}, 0 \leq p, q \leq 1$, and consider the subspaces of $\mathfrak{m}$ :

$$
\begin{aligned}
& \mathcal{V}_{1}=\mathbb{R}\left\{U_{\alpha}^{a} \mid \alpha \in \Delta_{1,1}^{+} ; a=0,1\right\}, \\
& \mathcal{V}_{2}=\mathbb{R}\left\{U_{\alpha}^{a} \mid \alpha \in \Delta_{1,0}^{+} ; a=0,1\right\}, \\
& \mathcal{V}_{3}=\mathbb{R}\left\{U_{\alpha}^{a} \mid \alpha \in \Delta_{0,1}^{+} ; a=0,1\right\} .
\end{aligned}
$$

Then $\mathfrak{m}=\mathcal{V}_{1} \oplus \mathcal{V}_{2} \oplus \mathcal{V}_{3}$ is a $B$-orthogonal decomposition as isotropy-irreducible subspaces stable under $J$, satisfying ([12])

$$
\left[\mathcal{V}_{k}, \mathcal{V}_{k}\right] \subset \mathfrak{k}, \quad k=1,2,3 ; \quad\left[\mathcal{V}_{k}, \mathcal{V}_{r}\right] \subset \mathcal{V}_{s},(k, r, s) \text { a permutation of }(1,2,3) .
$$


Theorem 5.4. Let $M=G / K$ be a 3-symmetric coset space of type $A_{3} I I$. For any homogeneous Riemannian metric $g$ on $M$, the $G$-invariant distribution associated to each $\mathcal{V}_{k} \subset \mathfrak{m}, k=1,2,3$, determines a harmonic map $\sigma_{k}:(M, g) \rightarrow$ $\left(G_{q_{k}}(M), g^{K}\right)$, where $q_{k}=\operatorname{dim} \mathcal{V}_{k}$. Moreover, $\sigma_{k}: M \rightarrow\left(G_{q_{k}}(M), g^{K}\right)$ is a minimal immersion. The corresponding transversally symmetric fibrations are:

(i) $\mathbb{C} G_{r_{i}, r_{j}} \rightarrow \frac{S U(n)}{S\left(U\left(r_{1}\right) \times U\left(r_{2}\right) \times U\left(r_{3}\right)\right)} \rightarrow \mathbb{C} G_{r_{k}, n-r_{k}}$, where $r_{i} \geq 1, r_{1}+r_{2}+r_{3}=n$, and $(i, j, k)$ is a cyclic permutation of $(1,2,3)$;

(ii) $\mathbb{C} P^{n-1} \rightarrow \frac{S O(2 n)}{U(n-1) \times S O(2)} \rightarrow \frac{S O(2 n)}{U(n)}, n \geq 4$;

(iii) $\frac{S O(2(n-1))}{U(n-1)} \rightarrow \frac{S O(2 n)}{U(n-1) \times S O(2)} \rightarrow \mathbb{R} G_{2(n-1), 2}, n \geq 4$;

(iv) $\mathbb{R} G_{2,8} \rightarrow \frac{E_{6}}{S O(8) \times S O(2) \times S O(2)} \rightarrow \frac{E_{6}}{S O(10) \times S O(2)}$.

Proof. Put $\mathfrak{p}=\mathcal{V}_{k}$, for $k=1,2,3$, and $\mathfrak{n}=\mathcal{V}_{r} \oplus \mathcal{V}_{s}$. ¿From $(5.3)$, we have $[\mathfrak{p}, \mathfrak{p}] \subset \mathfrak{k}$, $[\mathfrak{n}, \mathfrak{n}] \subset \mathfrak{p}$ and $[\mathfrak{p}, \mathfrak{n}] \subset \mathfrak{n}$. Then, using Lemma 4.1, the G-invariant distribution determined by $\mathfrak{p}$ is integrable and the foliation is transversally symmetric for any $G$ invariant metric on $M$. From Theorem 4.2, it is a harmonic totally geodesic foliation and a minimal immersion into its Grassmannian bundle. The irreducibility of $\mathcal{V}_{k}$ implies that each $G$-invariant metric on $M=G / K$ is determined by an $\operatorname{Ad}(K)$ invariant inner product of the form $\langle\cdot, \cdot\rangle=\sum_{r=1}^{3} \lambda_{r} B_{\mid \mathcal{V}_{r}}$ on $\mathfrak{m}$, for $\lambda_{r}>0, r=$ $1,2,3$, and its corresponding homogeneous structure $S$ satisfies

$$
S_{\mathcal{V}_{k}} \mathcal{V}_{k}=0, \quad S_{\mathcal{V}_{k}} \mathcal{V}_{r} \subset \mathcal{V}_{s}
$$

where $(k, r, s)$ is a permutation of $(1,2,3)$. From this and $(4.5)$, since $\xi_{\mathfrak{p}}=0$ and $\xi_{\mathfrak{n}}=S_{\mathfrak{n}}$,

$$
R_{\sigma}(U)=\left\langle\xi_{E_{i}} E_{a}, R_{U E_{i}} E_{a}\right\rangle=\left\langle S_{E_{q+j}} E_{a}, R_{U E_{q+j}} E_{a}\right\rangle=\left\langle S_{E_{q+j}} E_{a}, S_{\left[U, E_{q+j}\right]_{\mathfrak{m}}} E_{a}\right\rangle,
$$

for all $U \in \mathfrak{p}$. Then, using (5.3) and (5.4) and taking $\left\{E_{q+1}, \ldots, E_{n}\right\}$ as a $\langle\cdot, \cdot\rangle-$ orthonormal basis of $\mathfrak{n}$ adapted to the decomposition $\mathfrak{n}=\mathcal{V}_{r} \oplus \mathcal{V}_{s}$ we obtain $R_{\sigma}(U)=0$. Because for any transversally symmetric $G$-invariant foliation, $R_{\sigma}$ vanishes on $\mathfrak{n}$, it follows that $R_{\sigma}=0$ and then, by Theorem 4.2, $\sigma$ is a harmonic map.

If $\theta$ is of type $A_{3} I I$, the complex simple Lie algebra $\mathfrak{g}_{\mathbb{C}}$ is $\mathfrak{a}_{n-1}(n \geq 3), \mathfrak{d}_{n}$ $(n \geq 4)$ or $\mathfrak{e}_{6}$. On $\mathfrak{a}_{n-1}$, a set $\Delta^{+}$of positive roots is given by $\Delta^{+}=\left\{\alpha_{p, q}=\right.$ $\left.\alpha_{p}+\cdots+\alpha_{q} \mid 1 \leq p \leq q \leq n-1\right\}$. Then, for $\theta=\operatorname{Ad} d_{\exp 2 \pi \sqrt{-1} H}$, with $H=$ $\frac{1}{3}\left(H_{i}+H_{j}\right), 1<i<j<n-1$, we obtain $\Delta^{+}(H)=\Delta_{1}^{+}(H) \cup \Delta_{2}^{+}(H) \cup \Delta_{3}^{+}(H)$ where $\Delta_{1}^{+}(H)=\left\{\alpha_{p, q} \mid 1 \leq p \leq q<i\right\}, \Delta_{2}^{+}(H)=\left\{\alpha_{p, q} \mid i<p \leq q<j\right\}$ and $\Delta_{3}^{+}(H)=\left\{\alpha_{p, q} \mid j<p \leq q \leq n-1\right\}$. Then $\mathfrak{k}$ is of type $\mathfrak{a}_{i-1} \oplus \mathfrak{a}_{j-i-1} \oplus \mathfrak{a}_{n-1-j} \oplus \mathfrak{T}^{2}$. Hence $M$ is the quotient manifold $S U(n) / S(U(i) \times U(j-i) \times U(n-j))$.

On $\mathfrak{m}=\sum_{\alpha \in \Delta^{+} \backslash \Delta^{+}(H)} \mathbb{R} U_{\alpha}^{a}$, we consider the orthogonal decomposition $\mathfrak{m}=$ $\mathcal{V}_{1} \oplus \mathcal{V}_{2} \oplus \mathcal{V}_{3}$, given in $(5.2)$, where $\Delta_{1,1}^{+}=\left\{\alpha_{p, q} \mid p \leq i<j \leq q\right\}, \Delta_{1,0}^{+}=\left\{\alpha_{p, q} \mid\right.$ 
$1 \leq p \leq i \leq q<j\}$ and $\Delta_{0,1}^{+}=\left\{\alpha_{p, q} \mid i<p \leq j \leq q \leq n-1\right\}$. Then, putting $\mathfrak{l}_{k}=\mathfrak{k} \oplus \mathcal{V}_{k}, k=1,2,3$, it follows that $\mathfrak{l}_{k}$ can be written as $\mathfrak{l}_{k}=\mathfrak{l}_{k}^{1} \oplus \mathfrak{l}_{k}^{2}$, where

$$
\begin{aligned}
& \mathfrak{l}_{1}=\left(\sum_{\alpha \in\left\{\alpha_{i}, \ldots, \alpha_{j-1}\right\}} \mathbb{R} \sqrt{-1} H_{\alpha}+\sum_{\alpha \in \Delta_{2}^{+}(H)} \mathbb{R} U_{\alpha}^{a}\right) \\
& \oplus\left(\sum_{\alpha \in \Delta_{1,1}^{+}} \mathbb{R} \sqrt{-1} H_{\alpha}+\sum_{\alpha \in \Delta_{1,1}^{+} \cup \Delta_{1}^{+}(H) \cup \Delta_{3}^{+}(H)} \mathbb{R} U_{\alpha}^{a}\right), \\
& \mathfrak{l}_{2}=\left(\sum_{\alpha \in \Delta_{1,0}^{+}} \mathbb{R} \sqrt{-1} H_{\alpha}+\sum_{\alpha \in \Delta_{1,0}^{+} \cup \Delta_{1}^{+}(H) \cup \Delta_{2}^{+}(H)} \mathbb{R} U_{\alpha}^{a}\right) \\
& \oplus\left(\sum_{\alpha \in\left\{\alpha_{j}, \ldots, \alpha_{n-1}\right\}} \mathbb{R} \sqrt{-1} H_{\alpha}+\sum_{\alpha \in \Delta_{3}^{+}(H)} \mathbb{R} U_{\alpha}^{a}\right), \\
& \mathfrak{l}_{3}=\left(\sum_{\alpha \in \Delta_{0,1}^{+}} \mathbb{R} \sqrt{-1} H_{\alpha}+\sum_{\alpha \in \Delta_{0,1}^{+} \cup \Delta_{2}^{+}(H) \cup \Delta_{3}^{+}(H)} \mathbb{R} U_{\alpha}^{a}\right) \\
& \oplus\left(\sum_{\alpha \in\left\{\alpha_{1}, \ldots, \alpha_{i}\right\}} \mathbb{R} \sqrt{-1} H_{\alpha}+\sum_{\alpha \in \Delta_{1}^{+}(H)} \mathbb{R} U_{\alpha}^{a}\right) .
\end{aligned}
$$

Therefore $\mathfrak{l}_{2} \cong \mathfrak{s u}(j) \oplus \mathfrak{s u}(n-j) \oplus \mathfrak{T}^{1}$ and $\mathfrak{l}_{3} \cong \mathfrak{s u}(n-i) \oplus \mathfrak{s u}(i) \oplus \mathfrak{T}^{1}$. Moreover, it is clear that $\mathfrak{l}_{1}^{1} \cong \mathfrak{s u}(j-i) \oplus \mathfrak{T}^{1}$. Next, we show that $\mathfrak{l}_{1}^{2}$ is isomorphic to $\mathfrak{s u}(n+i-j)$. Denote by $\pi_{n-1+i-j}$ a system of simple roots $\pi_{n-1+i-j}=$ $\left\{\beta_{1}, \ldots, \beta_{n-1+i-j}\right\}$ of $\mathfrak{a}_{n-1+i-j}$. Let $\phi$ be the map $\phi\left(\beta_{1}\right)=\alpha_{1}, \ldots, \phi\left(\beta_{i-1}\right)=\alpha_{i-1}$, $\phi\left(\beta_{i}\right)=\alpha_{i, j}, \phi\left(\beta_{i+1}\right)=\alpha_{j+1}, \ldots, \phi\left(\beta_{n-1+i-j}\right)=\alpha_{n-1}$ between $\pi_{n-1+i-j}$ and $\left\{\alpha_{1}, \ldots, \alpha_{i-1}, \alpha_{i, j}, \alpha_{j+1}, \ldots \alpha_{n-1}\right\}$. Then $\phi$ can be extended by linearity to a bijection from $\Delta_{\mathfrak{a}_{n-1+i-j}}^{+}$to $\Delta_{1,1}^{+} \cup \Delta_{1}^{+}(H) \cup \Delta_{3}^{+}(H)$, where $\Delta_{\mathfrak{a}_{n-1+i-j}}^{+}$is the positive root set of $\mathfrak{a}_{n-1+i-j}$ generated by $\pi_{n-1+i-j}$. It gives the isomorphism, also denoted by $\phi$, between $\mathfrak{l}_{1}^{2}$ and $\mathfrak{s u}(n+i-j)$ by setting $\phi\left(\sqrt{-1} H_{\beta_{s}}\right)=\sqrt{-1} H_{\phi\left(\beta_{s}\right)}$ and $\phi\left(U_{\beta_{s}}^{a}\right)=U_{\phi\left(\beta_{s}\right)}^{a}$, for $s=1, \ldots, n-1+i-j$.

Taking $r_{1}=i, r_{2}=j-i$ and $r_{3}=n-j$, we have the fibrations given in (i).

On $\mathfrak{d}_{n}(n \geq 4)$,

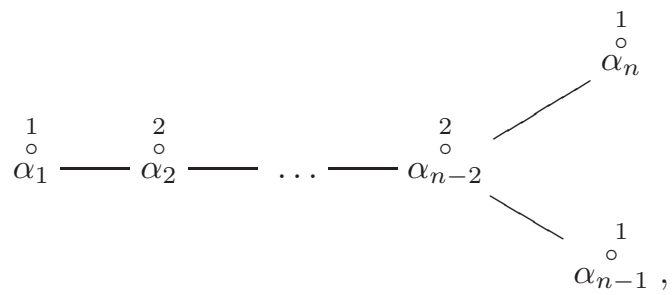

the automorphism $\theta$ of type $A_{3} I I$ is determined by $H=\frac{1}{3}\left(H_{n-1}+H_{n}\right)$. The other possibilities for $H$, i.e., $H=\frac{1}{3}\left(H_{n}+H_{n-1}\right)$ or $H=\frac{1}{3}\left(H_{1}+H_{n}\right)$, give automorphisms conjugate to the first one. Here, a set $\Delta^{+}$of positive roots is given by

$$
\begin{aligned}
\Delta^{+}=\{ & \alpha_{p}(1 \leq p \leq n), \alpha_{p, q}(1 \leq p<q<n), \tilde{\alpha}_{p, q}=\alpha_{p, n-2}+\alpha_{q, n} \\
& (1 \leq p<q \leq n, p \leq n-2)\} .
\end{aligned}
$$

Then $\Delta^{+}(H)=\left\{\alpha_{p, q}(1 \leq p \leq q \leq n-2)\right\}$ and $\mathfrak{k}$ is of type $\mathfrak{a}_{n-2} \oplus \mathfrak{T}^{2}$. Hence $M$ is the quotient manifold $S O(2 n) / U(n-1) \times S O(2)$. Next, we consider the orthogonal decomposition $\mathfrak{m}=\mathcal{V}_{1} \oplus \mathcal{V}_{2} \oplus \mathcal{V}_{3}$ where the corresponding subsets of positive roots are given by

$$
\begin{aligned}
& \Delta_{1,1}^{+}=\left\{\tilde{\alpha}_{p, q} \mid 1 \leq p<q \leq n-1\right\}, \quad \Delta_{1,0}^{+}=\left\{\alpha_{p, n-1} \mid 1 \leq p \leq n-1\right\} \\
& \Delta_{0,1}^{+}=\left\{\alpha_{n}, \tilde{\alpha}_{p, n} \mid 1 \leq p \leq n-2\right\} .
\end{aligned}
$$


Putting again $\mathfrak{l}_{k}=\mathfrak{k} \oplus \mathcal{V}_{k}, k=1,2,3$, we have:

$$
\begin{aligned}
\mathfrak{l}_{1} & =\left(\sum_{\alpha \in \Delta_{1,1}^{+}} \mathbb{R} \sqrt{-1} H_{\alpha}+\sum_{\alpha \in \Delta_{1,1}^{+} \cup \Delta^{+}(H)} \mathbb{R} U_{\alpha}^{a}\right) \oplus \mathbb{R} \sqrt{-1} H_{\alpha_{n}} \\
& \cong \mathfrak{s o}(2(n-1)) \oplus \mathfrak{T}^{1}, \\
\mathfrak{l}_{2} & =\sum_{\alpha \in \pi} \mathbb{R} \sqrt{-1} H_{\alpha}+\sum_{\alpha \in \Delta_{1,0}^{+} \cup \Delta^{+}(H)} \mathbb{R} U_{\alpha}^{a} \cong \mathfrak{u}(n), \\
\mathfrak{l}_{3} & =\sum_{\alpha \in \pi} \mathbb{R} \sqrt{-1} H_{\alpha}+\sum_{\alpha \in \Delta_{0,1}^{+} \cup \Delta^{+}(H)} \mathbb{R} U_{\alpha}^{a} \cong \mathfrak{u}(n) .
\end{aligned}
$$

Here, the isomorphism between $\sum_{\alpha \in \Delta_{1,1}^{+}} \mathbb{R} \sqrt{-1} H_{\alpha}+\sum_{\alpha \in \Delta_{1,1}^{+} \cup \Delta^{+}(H)} \mathbb{R} U_{\alpha}^{a}$ and $\mathfrak{s o}(2(n-1))$ is obtained from the bijection $\phi: \pi_{n-1} \rightarrow\left\{\alpha_{1}, \ldots, \alpha_{n-2}, \tilde{\alpha}_{n-2, n-1}\right\}$, given by $\phi\left(\beta_{i}\right)=\alpha_{i}, i=1, \ldots, n-2$, and $\phi\left(\beta_{n-1}\right)=\tilde{\alpha}_{n-2, n-1}$, where $\pi_{n-1}=$ $\left\{\beta_{1}, \ldots, \beta_{n-1}\right\}$ is a simple system of positive roots for $\mathfrak{d}_{n-1}$. Then the fibrations (ii) and (iii) are determined; (ii) corresponds with the vertical distributions $\mathcal{V}_{2}$ and $\mathcal{V}_{3}$ and (iii) with $\mathcal{V}_{1}$

Finally, a system of positive roots $\Delta^{+}$on $\mathfrak{e}_{6}$,

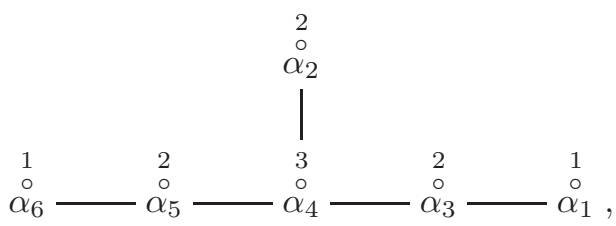

is given by

$$
\begin{aligned}
\Delta^{+}=\{ & \alpha_{1}, \alpha_{2} ; \alpha_{p, q}(3 \leq p \leq q \leq 6) ; \alpha_{1, p}, \alpha_{2, p}(4 \leq p \leq 6) ; \\
& \alpha_{1}+\alpha_{3, p}(3 \leq p \leq 6) ; \alpha_{2}+\alpha_{4, p}(4 \leq p \leq 6) ; \\
& \alpha_{4}+\alpha_{p, q}(p=1,2 ; q=5,6) ; \alpha_{1,6}+\alpha_{p, q}(3 \leq p<q \leq 5) ; \alpha_{2,6}+\alpha_{4,5}, \\
& \left.\alpha_{1,5}+\alpha_{3,4}, \alpha_{1,6}+\alpha_{3,5}+\alpha_{4}, \mu=\alpha_{1,6}+\alpha_{2,5}+\alpha_{4}\right\}
\end{aligned}
$$

and the automorphism $\theta$ of type $A_{3} I I$ is determined by $H=\frac{1}{3}\left(H_{1}+H_{6}\right)$. Hence we get

$$
\begin{aligned}
\Delta^{+}(H)= & \left\{\alpha_{2}, \alpha_{p, q}(3 \leq p \leq q \leq 5), \alpha_{2,4}, \alpha_{2,5}, \alpha_{2}+\alpha_{4}, \alpha_{2}+\alpha_{4,5}, \alpha_{4}+\alpha_{2,5}\right\}, \\
\Delta_{1,1}^{+}= & \left\{\alpha_{1,6}, \alpha_{1}+\alpha_{3,6}, \alpha_{4}+\alpha_{1,6}, \alpha_{1,6}+\alpha_{p, q}(3 \leq p<q \leq 5),\right. \\
& \left.\alpha_{1,6}+\alpha_{3,5}+\alpha_{4}, \mu\right\}, \\
\Delta_{1,0}^{+}= & \left\{\alpha_{1}, \alpha_{1, p}(p=4,5), \alpha_{1}+\alpha_{3, p}(3 \leq p \leq 5), \alpha_{4}+\alpha_{1,5}, \alpha_{1,5}+\alpha_{3,4}\right\}, \\
\Delta_{0,1}^{+}= & \left\{\alpha_{p, 6}(3 \leq p \leq 6), \alpha_{2,6}, \alpha_{2}+\alpha_{4,6}, \alpha_{4}+\alpha_{2,6}, \alpha_{4,5}+\alpha_{2,6}\right\} .
\end{aligned}
$$

Then the Lie subalgebras $\mathfrak{l}_{k}=\mathfrak{k} \oplus \mathcal{V}_{k}, k=1,2,3$, are

$$
\begin{aligned}
& \mathfrak{l}_{1}=\left(\sum_{\alpha \in \Delta_{1,1}^{+}} \mathbb{R} \sqrt{-1} H_{\alpha}+\sum_{\alpha \in \Delta_{1,1}^{+} \cup \Delta^{+}(H)} \mathbb{R} U_{\alpha}^{a}\right) \oplus \mathbb{R} \sqrt{-1} H_{\alpha_{6}}, \\
& \mathfrak{l}_{2}=\left(\sum_{\alpha \in \pi \backslash\left\{\alpha_{6}\right\}} \mathbb{R} \sqrt{-1} H_{\alpha}+\sum_{\alpha \in \Delta_{1,0}^{+} \cup \Delta^{+}(H)} \mathbb{R} U_{\alpha}^{a}\right) \oplus \mathbb{R} \sqrt{-1} H_{\alpha_{6}}, \\
& \mathfrak{l}_{3}=\left(\sum_{\alpha \in \pi \backslash\left\{\alpha_{1}\right\}} \mathbb{R} \sqrt{-1} H_{\alpha}+\sum_{\alpha \in \Delta_{0,1}^{+} \cup \Delta^{+}(H)} \mathbb{R} U_{\alpha}^{a}\right)+\mathbb{R} \sqrt{-1} H_{\alpha_{1}} .
\end{aligned}
$$


Using the corresponding Dynkin diagrams, one gets $\mathfrak{l}_{2} \cong \mathfrak{l}_{3} \cong \mathfrak{s o}(10) \oplus \mathfrak{T}^{1}$. Next we also show that $\sum_{\alpha \in \Delta_{1,1}^{+}} \mathbb{R} \sqrt{-1} H_{\alpha}+\sum_{\alpha \in \Delta_{1,1}^{+} \cup \Delta^{+}(H)} \mathbb{R} U_{\alpha}^{a}$ is isomorphic to $\mathfrak{s o}(10)$. Let $\Delta_{\mathfrak{d}_{5}}^{+}$be the positive root set for $\mathfrak{d}_{5}$ generated by a system of simple roots $\pi_{5}=\left\{\beta_{1}, \ldots, \beta_{5}\right\}$. Let $\phi$ be the bijection $\phi: \pi_{5} \rightarrow\left\{\alpha_{1}+\alpha_{3,6}, \alpha_{2}, \ldots, \alpha_{5}\right\}$, given by $\phi\left(\beta_{1}\right)=\alpha_{1}+\alpha_{3,6}, \phi\left(\beta_{2}\right)=\alpha_{2}, \phi\left(\beta_{3}\right)=\alpha_{4}, \phi\left(\beta_{4}\right)=\alpha_{5}$ and $\phi\left(\beta_{5}\right)=\alpha_{3}$. Then $\phi$ can be extended to $\Delta_{\mathfrak{d}_{5}} \rightarrow \Delta(H) \cup \Delta_{1,1}$ by linearity and the result follows as in the preceding cases. Hence, the fibration (iv) can be constructed.

Remark 5.5. Because the base space of these fibrations is an irreducible symmetric space of compact type, it must be isotropy irreducible and so, up to homotheties, it admits a unique invariant metric. Then, using Proposition 4.3, a fibration as in Proposition 5.4 with $\mathfrak{p}=\mathcal{V}_{k}$ and $\mathfrak{n}=\mathcal{V}_{r} \oplus \mathcal{V}_{s}, k=1,2,3$, is Riemannian if and only if the homogeneous metric on the 3 -symmetric space is determined by an inner product $\langle\cdot, \cdot\rangle=\sum_{i=1}^{3} \lambda_{i} B_{\mid \mathcal{V}_{i}}$, such that $\lambda_{r}=\lambda_{s}$.

Next suppose that $\theta$ has type $A_{3} I I I$. Then $m_{i}=2\left(H=\frac{2}{3} H_{i}\right)$, for some $i=1, \ldots, l$. Consider the subspaces of $\mathfrak{m}$ :

$$
\mathcal{H}=\mathbb{R}\left\{U_{\alpha}^{a} \mid \alpha \in \Delta_{1}^{+}, ; a=0,1\right\}, \quad \mathcal{V}=\mathbb{R}\left\{U_{\alpha}^{a} \mid \alpha \in \Delta_{2}^{+} ; a=0,1\right\},
$$

where $\Delta_{p}^{+}=\left\{\alpha \in \Delta^{+} \mid n_{i}(\alpha)=p\right\}, p=0,1,2$. Then $\Delta(H)=\Delta_{0}$ and $\mathfrak{m}=\mathcal{V} \oplus \mathcal{H}$ is an orthogonal decomposition of isotropy-irreducible subspaces stable under $J$, and satisfying, [12],

$$
[\mathcal{H}, \mathcal{H}]_{\mathfrak{m}} \subset \mathcal{V}, \quad[\mathcal{V}, \mathcal{V}] \subset \mathfrak{k}, \quad[\mathcal{V}, \mathcal{H}] \subset \mathcal{H}
$$

Theorem 5.6. Let $M=G / K$ be a 3-symmetric coset space of type $A_{3} I I I$. For any homogeneous Riemannian metric $g$ on $M$, the $G$-invariant distribution $\mathcal{V}$ determines a harmonic map $\sigma:(M, g) \rightarrow\left(G_{q}(M), g^{K}\right)$. Moreover, the immersion $\sigma: M \rightarrow\left(G_{q}(M), g^{K}\right)$ is minimal. The corresponding transversally symmetric (Riemannian) fibrations are:

$$
\begin{aligned}
& \text { (i) } \frac{S O(2 i)}{U(i)} \rightarrow \frac{S O(2 n+1)}{U(i) \times S O(2(n-i)+1)} \rightarrow \mathbb{R} G_{2 i, 2(n-i)+1},(n>2, i>1) \\
& \text { (ii) } \frac{S p(i)}{U(i)} \rightarrow \frac{S p(n)}{U(i) \times S p(n-i)} \rightarrow \mathbb{H} G_{i, n-i},(n \geq 2, i<n) ; \\
& \text { (iii) } \frac{S O(2 i)}{U(i)} \rightarrow \frac{S O(2 n)}{U(i) \times S O(2(n-i))} \rightarrow \mathbb{R} G_{2 i, 2(n-i)},(n \geq 4,2 \leq i \leq n-2) \text {; } \\
& \text { (iv) } S^{2} \rightarrow \frac{G_{2}}{U(2)} \rightarrow \frac{G_{2}}{S U(2) \times S U(2)} ; \\
& \text { (v) } S^{2} \rightarrow \frac{F_{4}}{S p(3) \times S O(2)} \rightarrow \frac{F_{4}}{S p(3) \times S U(2)} ; \\
& \text { (vi) } \mathbb{R} G_{2,7} \rightarrow \frac{F_{4}}{S O(7) \times S O(2)} \rightarrow \frac{F_{4}}{S O(9)} ; \\
& \text { (vii) } \mathbb{C} G_{1,5} \rightarrow \frac{E_{6}}{S(U(5) \times U(1)) \times S U(2)} \rightarrow \frac{E_{6}}{S U(6) \times S U(2)} \\
& \text { (viii) } S^{2} \rightarrow \frac{E_{6}}{S U(6) \times T^{1}} \rightarrow \frac{E_{6}}{S U(6) \times S U(2)} ;
\end{aligned}
$$


(ix) $S^{2} \rightarrow \frac{E_{7}}{S O(12) \times S O(2)} \rightarrow \frac{E_{7}}{S O(12) \times S U(2)}$;

(x) $\mathbb{C} G_{1,7} \rightarrow \frac{E_{7}}{S(U(7) \times U(1))} \rightarrow \frac{E_{7}}{S U(8)}$;

(xi) $\mathbb{R} G_{2,10} \rightarrow \frac{E_{7}}{S U(2) \times S O(10) \times S O(2)} \rightarrow \frac{E_{7}}{S O(12) \times S U(2)}$;

(xii) $S^{2} \rightarrow \frac{E_{8}}{E_{7} \times S O(2)} \rightarrow \frac{E_{8}}{E_{7} \times S U(2)}$;

(xiii) $\mathbb{R} G_{2,14} \rightarrow \frac{E_{8}}{S O(14) \times S O(2)} \rightarrow \frac{E_{8}}{S O(16)}$.

Proof. From (5.5), the distribution $\mathcal{V}$ is integrable and the $G$-invariant foliation is transversally symmetric. Moreover, by Proposition 5.2, it is Riemannian. So it follows from Theorem 4.2 that $\mathcal{V}$ determines a harmonic map and a minimal immersion.

If $\theta$ is of type $A_{3} I I I$, one gets that $\mathfrak{g}_{\mathbb{C}}$ is one of the following: $\mathfrak{b}_{n}(n \geq 2), \mathfrak{c}_{n}$ $(n \geq 2), \mathfrak{d}_{n}(n \geq 4), \mathfrak{g}_{2}, \mathfrak{f}_{4}, \mathfrak{e}_{6}, \mathfrak{e}_{7}$ and $\mathfrak{e}_{8}$.

In similar way as for type $A_{3} I I$, we can determine on these complex simple Lie algebras the sets of positive roots $\Delta^{+}$and $\Delta_{p}^{+}$, for $p=0,1,2$, and the Lie subalgebra $\mathfrak{l}=\mathfrak{k} \oplus \mathcal{V}$, which now can be expressed as $\mathfrak{l}=\mathfrak{h}+\sum_{\alpha \in \Delta_{0}^{+} \cup \Delta_{2}^{+}} \mathbb{R} U_{\alpha}^{a}$. Here, we only consider exceptional Lie algebras. This involves the fibrations (iv)-(xiii). For $\mathfrak{b}_{n}, \mathfrak{c}_{n}$ and $\mathfrak{d}_{n}$, the sets $\Delta^{+}$and $\Delta_{p}^{+}, p=0,1,2$, are described in [12].

For $\mathfrak{g}_{2}$,

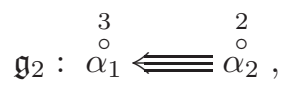

a set of positive roots is given by $\Delta^{+}=\left\{\alpha_{1}, \alpha_{2}, \alpha_{1}+\alpha_{2}, 2 \alpha_{1}+\alpha_{2}, 3 \alpha_{1}+\alpha_{2}\right.$, $\left.\mu=3 \alpha_{1}+2 \alpha_{2}\right\}$. In this case $H=\frac{2}{3} H_{2}, \Delta_{2}^{+}=\{\mu\}$ and $\Delta^{+}(H)=\left\{\alpha_{1}\right\}$. Then $\mathfrak{l}=\left(\mathbb{R} \sqrt{-1} H_{\mu} \oplus \mathbb{R} U_{\mu}^{a}\right) \oplus\left(\mathbb{R} \sqrt{-1} H_{\alpha_{1}} \oplus \mathbb{R} U_{\alpha_{1}}^{a}\right) \cong \mathfrak{s u}(2) \oplus \mathfrak{s u}(2)$. Hence $F$ is the 2-sphere $S^{2}$ and the fibration (iv) is obtained. On $\mathfrak{f}_{4}$,

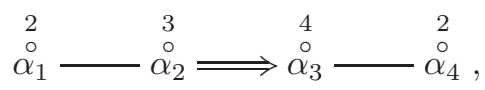

$\mu=2 \alpha_{1,4}+\alpha_{2,3}+\alpha_{3}$ and a set of positive roots is given by

$$
\begin{aligned}
\Delta^{+}=\{ & \alpha_{p, q}(1 \leq p \leq q \leq 4), \alpha_{2,3}+\alpha_{3}, \alpha_{1, q}+\alpha_{p, 3}(p=2,3 ; q=3,4), \\
& \alpha_{2,4}+\alpha_{3, q}(q=3,4), \alpha_{14}+\alpha_{p 4}(p=2,3), \alpha_{1,4}+\alpha_{2, q}+\alpha_{3}(q=3,4), \\
& \left.\alpha_{1,4}+\alpha_{2,4}+\alpha_{p, 3}+\alpha_{3}(p=2,3), \mu\right\} .
\end{aligned}
$$

Here $H=\frac{2}{3} H_{1}$ or $H=\frac{2}{3} H_{4}$. For $H=\frac{2}{3} H_{1}$, one gets

$$
\Delta^{+}(H)=\left\{\alpha_{p, q}(2 \leq p \leq q \leq 4), \alpha_{2}+2 \alpha_{3}, \alpha_{2}+2 \alpha_{3}+\alpha_{4}, \alpha_{2}+2 \alpha_{3}+2 \alpha_{4}\right\},
$$

which coincides with a positive root set for $\mathfrak{c}_{3}$. Then, $\Delta_{2}^{+}=\{\mu\}$ and we have

$$
\begin{aligned}
\mathfrak{l}=\left(\mathbb{R} \sqrt{-1} H_{\mu} \oplus \mathbb{R} U_{\mu}^{a}\right) \oplus\left(\mathbb{R}\left\{\sqrt{-1} H_{\alpha_{2}}, \sqrt{-1} H_{\alpha_{3}}, \sqrt{-1} H_{\alpha_{4}}\right\}\right. \\
\left.\oplus \sum_{\alpha \in \Delta^{+}(H)} \mathbb{R} U_{\alpha}^{a}\right) \cong \mathfrak{s u}(2) \oplus \mathfrak{s p}(3) .
\end{aligned}
$$


This gives the fibration (v). For $H=\frac{2}{3} H_{4}$, one gets

$$
\begin{aligned}
\Delta^{+}(H)= & \left\{\alpha_{p, q}(1 \leq p \leq q \leq 3), \alpha_{2}+2 \alpha_{3}, \alpha_{1}+\alpha_{2}+2 \alpha_{3}, \alpha_{1}+2 \alpha_{2}+2 \alpha_{3}\right\} \\
\Delta_{2}^{+}= & \left\{\alpha_{2,4}+\alpha_{3,4}, \alpha_{1,4}+\alpha_{p, 4}(p=2,3), \alpha_{1,4}+\alpha_{2,4}+\alpha_{3},\right. \\
& \left.\alpha_{1,4}+\alpha_{2,4}+\alpha_{p, 3}+\alpha_{3}(p=2,3), \mu\right\} .
\end{aligned}
$$

Putting $\beta_{1}=\alpha_{2,4}+\alpha_{3,4}, \beta_{2}=\alpha_{1}, \beta_{3}=\alpha_{2}$ and $\beta_{4}=\alpha_{3},\left\{\beta_{1}, \ldots, \beta_{4}\right\}$ is a system of simple roots of $\mathfrak{b}_{4}$ and the positive root set generated by it coincides with $\Delta^{+}(H) \cup \Delta_{2}^{+}$. Hence $\mathfrak{l} \cong \mathfrak{s o}(9)$ and we obtain (vi).

On $\mathfrak{e}_{6}, H=\frac{2}{3} H_{3}$ and $H=\frac{2}{3} H_{5}$ determine conjugate automorphisms of type $A_{3} I I I$. Also $H=\frac{2}{3} H_{2}$ determines an automorphism of type $A_{3} I I I$. We start with $H=\frac{2}{3} H_{3}$. Then

$$
\begin{aligned}
\Delta^{+}(H) & =\left\{\alpha_{1}, \alpha_{2}, \alpha_{p, q}(4 \leq p \leq q \leq 6), \alpha_{2}+\alpha_{4, p}(4 \leq p \leq 6)\right\}, \\
\Delta_{2}^{+} & =\left\{\alpha_{1,5}+\alpha_{3,4}, \alpha_{1,6}+\alpha_{3,4}, \alpha_{1,6}+\alpha_{3,5}, \alpha_{1,6}+\alpha_{3,5}+\alpha_{4}, \mu\right\} .
\end{aligned}
$$

Let $\Delta_{\mathfrak{a}_{5}}^{+}$be the positive root set for $\mathfrak{a}_{5}$ generated by a system of simple roots $\pi_{5}=\left\{\beta_{1}, \ldots, \beta_{5}\right\}$. Let $\phi$ be the bijection $\phi: \pi_{5} \rightarrow\left\{\alpha_{2}, \alpha_{4}, \alpha_{5}, \alpha_{6}, \alpha_{1,5}+\alpha_{3,4}\right\}$, given by $\phi\left(\beta_{1}\right)=\alpha_{2}, \phi\left(\beta_{2}\right)=\alpha_{4}, \phi\left(\beta_{3}\right)=\alpha_{5}, \phi\left(\beta_{4}\right)=\alpha_{6}$ and $\phi\left(\beta_{5}\right)=\alpha_{1,5}+\alpha_{3,4}$. Then $\phi$ can be extended by linearity to a homomorphism from $\mathfrak{s u}(6)$ to $\mathfrak{l}$ and one gets

$$
\mathfrak{l}=\left(\sum_{\alpha \in \tilde{\Delta}^{+}} \mathbb{R} U_{\alpha}^{a}+\sum_{\alpha \in \Delta_{2}^{+}} \mathbb{R} \sqrt{-1} H_{\alpha}\right) \oplus\left(\mathbb{R} \sqrt{-1} H_{\alpha_{1}} \oplus \mathbb{R} U_{\alpha_{1}}^{a}\right) \cong \mathfrak{s u}(6) \oplus \mathfrak{s u}(2) .
$$

Hence we get the fibration given in (vii). Next, we consider $H=\frac{2}{3} H_{2}$. This implies that

$$
\Delta^{+}(H)=\left\{\alpha_{1}, \alpha_{p, q}(3 \leq p \leq q \leq 6), \alpha_{1}+\alpha_{3, p}(3 \leq p \leq 6)\right\}
$$

and $\Delta_{2}^{+}=\{\mu\}$. Since $\Delta^{+}(H)$ is a root set for $\mathfrak{a}_{5}$ and $\left\{\alpha_{1}, \alpha_{3}, \ldots, \alpha_{6}\right\}$ a simple system, we have $\mathfrak{l} \cong \mathfrak{s u}(6) \oplus \mathfrak{s u}(2)$. This gives the fibration in (viii).

On $\mathfrak{e}_{7}$,

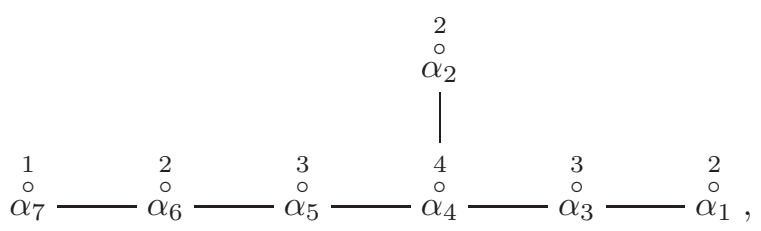

a positive system of roots is given by

$$
\begin{aligned}
\Delta^{+}=\{ & \alpha_{1}, \alpha_{2} ; \alpha_{p, q}(3 \leq p \leq q \leq 7) ; \alpha_{1, p}, \alpha_{2, p}(4 \leq p \leq 7) ; \alpha_{1}+\alpha_{3, p}(3 \leq p \leq 7) ; \\
& \alpha_{2}+\alpha_{4, p}(4 \leq p \leq 7) ; \alpha_{4}+\alpha_{p, q}(p=1,2 ; q=5,6,7) ; \alpha_{1,6}+\alpha_{p, q} \\
& (3 \leq p<q \leq 5) ; \alpha_{2, p}+\alpha_{4,5}(p=6,7), \alpha_{4,6}+\alpha_{2,7}, \alpha_{1,7}+\alpha_{p, 6}(p=3,4), \\
& \alpha_{1,7}+\alpha_{p, q}(3 \leq p<q \leq 5), \alpha_{1,5}+\alpha_{3,4}, \alpha_{1,6}+\alpha_{p, 5}+\alpha_{4}(p=2,3), \\
& \alpha_{1,7}+\alpha_{p, q}+\alpha_{4}(p=2,3 ; q=5,6), \alpha_{1,7}+\alpha_{p, 6}+\alpha_{4,5}(p=2,3), \\
& \left.\alpha_{1,7}+\alpha_{2,6}+\alpha_{p, 5}+\alpha_{4}(p=3,4), \mu\right\} .
\end{aligned}
$$


The possible automorphisms of type $A_{3} I I I$ are determined by $H=\frac{2}{3} H_{1}, H=\frac{2}{3} H_{2}$ or $H=\frac{2}{3} H_{6}$. If $H=\frac{2}{3} H_{1}$, then

$$
\begin{aligned}
\Delta^{+}(H)=\{ & \alpha_{2} ; \alpha_{p, q}(3 \leq p \leq q \leq 7) ; \alpha_{2, p}(4 \leq p \leq 7) ; \alpha_{2}+\alpha_{4, p}(4 \leq p \leq 7) \\
& \left.\alpha_{4}+\alpha_{2, q}(q=5,6,7) ; \alpha_{2, p}+\alpha_{4,5}(p=6,7), \alpha_{4,6}+\alpha_{2,7}\right\}
\end{aligned}
$$

and $\Delta_{2}^{+}=\{\mu\}$. Using Dynkin diagrams, $\Delta^{+}(H)$ is a root set for $\mathfrak{d}_{6}$ with simple system $\left\{\beta_{1}, \ldots, \beta_{6}\right\}$, where $\beta_{k}=\alpha_{8-k}, k=1, \ldots, 6$. Hence, $\mathfrak{l} \cong \mathfrak{s o}(12) \oplus \mathfrak{s u}(2)$. This gives the fibration in (ix). If $H=\frac{2}{3} H_{2}$, then

$$
\begin{gathered}
\Delta^{+}(H)=\left\{\alpha_{1}, \alpha_{p, q}(3 \leq p \leq q \leq 7) ; \alpha_{1}+\alpha_{3, p}(3 \leq p \leq 7)\right\}, \\
\Delta_{2}^{+}=\left\{\alpha_{1,6}+\alpha_{2,5}+\alpha_{4}, \alpha_{1,7}+\alpha_{2, q}+\alpha_{4}(q=5,6), \alpha_{1,7}+\alpha_{2,6}+\alpha_{4,5},\right. \\
\left.\alpha_{1,7}+\alpha_{2,6}+\alpha_{p, 5}+\alpha_{4}(p=3,4), \mu\right\} .
\end{gathered}
$$

Put $\beta_{1}=\alpha_{1}, \beta_{2}=\alpha_{3}, \beta_{3}=\alpha_{4}, \beta_{4}=\alpha_{5}, \beta_{5}=\alpha_{6}, \beta_{6}=\alpha_{7}$ and $\beta_{7}=\alpha_{1,6}+\alpha_{2,5}$ $+\alpha_{4}$. Then $\left\{\beta_{1}, \ldots, \beta_{7}\right\}$ is a system of simple roots of $\mathfrak{a}_{7}$ and the positive root set generated by them coincides with $\Delta^{+}(H) \cup \Delta_{2}^{+}$. Hence $\mathfrak{l} \cong \mathfrak{s u}(8)$ and we obtain $(\mathrm{x})$. If $H=\frac{2}{3} H_{6}$, then

$$
\begin{aligned}
\Delta^{+}(H)= & \left\{\alpha_{1}, \alpha_{2} ; \alpha_{p, q}(3 \leq p \leq q \leq 5) ; \alpha_{7}, \alpha_{1, p}, \alpha_{2, p}(p=4,5) ; \alpha_{1}+\alpha_{3, p}\right. \\
& \left.(p=3,4,5) ; \alpha_{2}+\alpha_{4, p}(p=4,5) ; \alpha_{4}+\alpha_{p, 5}(p=1,2) ; \alpha_{1,5}+\alpha_{3,4}\right\}, \\
\Delta_{2}^{+}= & \left\{\alpha_{2,7}+\alpha_{4,6}, \alpha_{1,7}+\alpha_{p, 6}(p=3,4), \alpha_{1,7}+\alpha_{p, 6}+\alpha_{4}(p=2,3),\right. \\
& \left.\alpha_{1,7}+\alpha_{p, 6}+\alpha_{4,5}(p=2,3), \alpha_{1,7}+\alpha_{2,6}+\alpha_{p, 5}+\alpha_{4}(p=3,4), \mu\right\} .
\end{aligned}
$$

Let $\Delta_{\mathfrak{d}_{6}}^{+}$be the positive root set for $\mathfrak{d}_{6}$ generated by a system of simple roots $\pi_{6}=\left\{\beta_{k} \mid k=1, \ldots 6\right\}$. Let $\phi$ be the bijection $\phi: \pi_{6} \rightarrow\left\{\alpha_{2,7}+\alpha_{4,6}, \alpha_{1}, \ldots, \alpha_{5}\right\}$, given by $\phi\left(\beta_{1}\right)=\alpha_{2,7}+\alpha_{4,6}, \phi\left(\beta_{2}\right)=\alpha_{1}, \phi\left(\beta_{3}\right)=\alpha_{3}, \phi\left(\beta_{4}\right)=\alpha_{4}, \phi\left(\beta_{5}\right)=\alpha_{2}$ and $\phi\left(\beta_{6}\right)=\alpha_{5}$. Then $\phi$ can be extended by linearity to $\Delta_{\mathfrak{d}_{6}}^{+} \rightarrow \tilde{\Delta}^{+}=\Delta_{2}^{+} \cup\left(\Delta^{+}(H) \backslash\right.$ $\left.\left\{\alpha_{7}\right\}\right)$ and so,

$$
\mathfrak{l}=\left(\sum_{\alpha \in \tilde{\Delta}^{+}} \mathbb{R} U_{\alpha}^{a}+\sum_{\alpha \in \Delta_{2}^{+}} \mathbb{R} \sqrt{-1} H_{\alpha}\right) \oplus\left(\mathbb{R} \sqrt{-1} H_{\alpha_{7}} \oplus \mathbb{R} U_{\alpha_{7}}^{a}\right) \cong \mathfrak{s o}(12) \oplus \mathfrak{s u}(2)
$$

Thus we get the fibration (xi). On $\mathfrak{e}_{8}$,

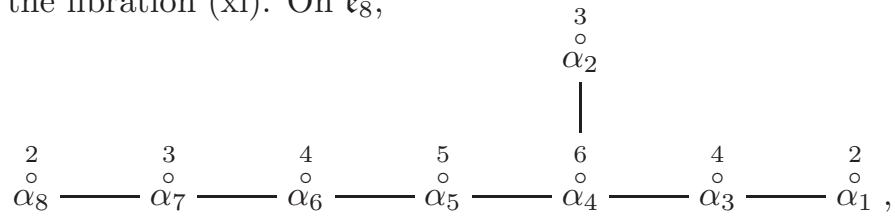

we consider the automorphisms $\theta$ of type $A_{3} I I I$ determined by $H=\frac{2}{3} H_{1}$ and $H=\frac{2}{3} H_{8}$. If $H=\frac{2}{3} H_{8}, \Delta^{+}(H)$ is the set of positive roots of $\mathfrak{e}_{7}$ and $\Delta^{+}=$ $\Delta^{+}(H) \cup\{\mu\} \cup\{\alpha, \mu-\alpha\}$, where $\mu=2 \alpha_{1,8}+\alpha_{2,7}+\alpha_{3,6}+\alpha_{4,5}+\alpha_{4}$ and $\alpha$ is any element of the following set:

$$
\begin{gathered}
\left\{\alpha_{p, 8}(1 \leq p \leq 8), \alpha_{1,8}+\alpha_{3, q}, \alpha_{1,8}+\alpha_{4, q}, \alpha_{2,8}+\alpha_{4, q}(4 \leq q \leq 7),\right. \\
\alpha_{3,8}+\alpha_{1}, \alpha_{4,8}+\alpha_{2}, \alpha_{1,8}+\alpha_{3, q}+\alpha_{4}(5 \leq q \leq 7), \\
\left.\alpha_{1,8}+\alpha_{2, q}+\alpha_{4}(5 \leq q \leq 6), \alpha_{1,8}+\alpha_{2,6}+\alpha_{4,5}\right\} .
\end{gathered}
$$


Since $\Delta_{2}^{+}=\{\mu\}, \mathfrak{l} \cong \mathfrak{e}_{7} \oplus \mathfrak{s u}(2)$. This gives the fibration (xii). If $H=\frac{2}{3} H_{1}$, we have

$$
\begin{aligned}
\Delta^{+}(H)=\{ & \alpha_{2}, \alpha_{p, q}(3 \leq p \leq q \leq 8), \alpha_{2, p}(4 \leq p \leq 8), \alpha_{4}+\alpha_{2, p}(5 \leq p \leq 8), \\
& \alpha_{2}+\alpha_{4, p}(4 \leq p \leq 8), \alpha_{2, p}+\alpha_{4,5}(6 \leq p \leq 8), \\
& \left.\alpha_{2, p}+\alpha_{4,6}(p=7,8), \alpha_{2,8}+\alpha_{4,7}\right\} \\
\Delta_{2}^{+}=\{ & \mu, \mu-\alpha\}
\end{aligned}
$$

where $\alpha$ is any element of

$$
\left\{\alpha_{2}, \alpha_{p, q}(3 \leq p \leq q \leq 8), \alpha_{2, p}(4 \leq p \leq 8), \alpha_{2}+\alpha_{4,8}, \alpha_{2,8}+\alpha_{4, p}(4 \leq p \leq 8)\right\} .
$$

Put $\beta_{1}=\mu-\left(\alpha_{2,8}+\alpha_{4,8}\right)=\alpha_{1,7}+\alpha_{1,6}+\alpha_{3,5}+\alpha_{4}$ and $\beta_{k}=\alpha_{10-k}$, for $k \in$ $\{2, \ldots, 8\}$. Then $\left\{\beta_{1}, \ldots, \beta_{8}\right\}$ is a system of simple roots of $\mathfrak{d}_{8}$ and the positive root set generated by it coincides with $\Delta^{+}(H) \cup \Delta_{2}^{+}$. Hence $\mathfrak{l} \cong \mathfrak{s o}(16)$ and we obtain the fibration (xiii).

\section{References}

[1] Besse, A. L.: Einstein manifolds. Ergebnisse der Mathematik und ihrer Grenzgebiete (3) 10, Springer-Verlag, Berlin, 1987.

[2] Blair, D. E.: Riemannian geometry of contact and symplectic manifolds. Progress in Mathematics 203, Birkhäuser Boston, Boston, MA, 2002.

[3] Cleyton, R. and Swann, A. F.: Einstein metrics via intrinsic or parallel torsion. Math. Z. 247 (1004), no. 3, 513-528.

[4] Chacón, P. M., Naveira, A. M. and Weston, J. M.: On the energy of distributions, with application to the quaternionic Hopf fibrations. Monatsh. Math. 133 (2001), 281-294.

[5] Choi, B.-Y. And Yim, J.-W.: Distributions on Riemannian manifolds, which are harmonic maps. Tôhoku Math. J. 55 (2003), 175-188.

[6] Eells, J. And Lemaire, L.: A report on harmonic maps. Bull. London Math. Soc. 10 (1978), 1-68.

[7] Eells, J. And Lemaire, L.: Another report on harmonic maps. Bull. London Math. Soc. 20 (1988), 385-524.

[8] Gil-Medrano, O., González-Dávila, J. C. and Vanhecke, L.: Harmonicity and minimality of oriented distributions. Israel J. Math. 143 (2004), 253-279.

[9] GonzÁlez-DÁvila, J. C.: Isotropic Jacobi fields on compact 3-symmetric spaces. J. Differential Geom. 83 (2009), 273-288.

[10] González-Dávila, J.Ċ. And Martín Cabrera, F.: Harmonic G-structures. Math. Proc. Cambridge Philos. Soc. 146 (2009), 435-459.

[11] GonzÁlez-DÁvila, J.Ċ. AND Martín CABrera, F.: Harmonic almost contact structures via the intrinsic torsion. Israel J. Math. 181 (2011), 145-187.

[12] González-Dávila, J.Ċ. And Martín Cabrera, F.: Homogeneous nearly Kähler manifolds. Ann. Glob. Anal. Geom. 42 (2012), 147-170. 
[13] González-Dávila, J. C. And Naveira, A. M.: Existence of non-isotropic conjugate points on rank one normal homogeneous spaces. Ann. Glob. Anal. Geom. 45 (2014), no. 3, 211-231.

[14] Gray, A.: Riemannian manifolds with geodesic symmetries of order 3. J. Differential Geom. 7 (1972), 343-369.

[15] Helgason, S.: Differential geometry, Lie groups, and symmetric spaces. Pure and Applied Mathematics 80, Academic Press, New York-London, 1978.

[16] Kobayashi, S. And Nomizu, K.: Foundations of differential geometry. I, II. Interscience Publishers, New York, 1963, 1969.

[17] Lawson, H. B. And Michelsohn, M. L.: Spin geometry. Princeton Mathematical Series 38, Princeton University Press, 1989.

[18] Nomizu, K.: Invariant affine connections on homogeneous spaces. Amer. J. Math. 76 (1954), 33-65.

[19] Onishchik, A. L.: Transitive compact transformation groups. Mat. Sb. (N.S.) 60 (1963), 447-485 (Russian); English traslation in Amer. Math. Soc. Transl. 55 (1966), 153-194.

[20] Ornea, L. and Vanhecke, L.: Harmonicity and minimality of vector fields and distributions on locally conformal Kähler and hyperkähler manifolds. Bull. Belg. Math. Soc. Simon Stevin 12 (2005), 543-555.

[21] Tondeur, P. And Vanhecke, L.: Transversally symmetric Riemannian foliations. Tôhoku Math. J. 42 (1990), 307-317.

[22] Turgut Vanli, A. And Blair, D. E.: The Boothby-Wang fibration of the Iwasawa manifold as a critical point of the energy. Monatsh. Math. 147 (2006), 75-84.

[23] Stepanov, S. E.: Riemannian almost product manifolds and submersions. Part I. J. Math. Sci. (New York) 99 (2000), no. 6, 1788-1810.

[24] Tricerri, F. And VAnhecke, L.: Homogeneous structures on Riemannian manifolds. London Mathematical Society Lecture Note Series 83, Cambridge University Press, Cambridge, 1983.

[25] Urakawa, H.: Calculus of variations and harmonic maps. Translations of Mathematical Monographs 132, American Mathematical Society, Providence, RI, 1993.

[26] Wilking, B.: The normal homogeneous space $(S U(3) \times S O(3)) / U^{\bullet}(2)$ has positive sectional curvature. Proc. Amer. Math. Soc. 127 (1999), no. 4, 1191-1194.

[27] Wolf, J. A. And Gray, A.: Homogeneous spaces defined by Lie group automorphisms. I, II. J. Diff. Geom. 2 (1968), 77-159.

[28] Wood, C. M.: A class of harmonic almost-product structures. J. Geom. Physics 14 (1994), 25-42.

[29] Wood, C. M.: Harmonic sections of homogeneous fibre bundles. Diff. Geom. Appl. 19 (2003), 193-210.

Received April 23, 2012.

José Carmelo González-DÁvila: Department of Fundamental Mathematics and Statistics, University of La Laguna, 38200 La Laguna, Tenerife, Spain.

E-mail: jcgonza@ull.es

Supported by DGI (Spain) and FEDER Project MTM2010-15444. 